\title{
Myofibroblastic Conversion and Regeneration of Mesothelial Cells in Peritoneal and Liver Fibrosis
}

\author{
Ingrid Lua, * Yuchang Li, ${ }^{*}$ Lamioko S. Pappoe, ${ }^{\dagger}$ and Kinji Asahina*
}

From the Department of Pathology, * Southern California Research Center for Alcoholic Liver and Pancreatic Diseases (ALPD) and Cirrhosis, Keck School of Medicine, University of Southern California, Los Angeles; and the Division of Nephrology, ${ }^{\dagger}$ Los Angeles County+University of Southern California Medical Center, Los Angeles, California

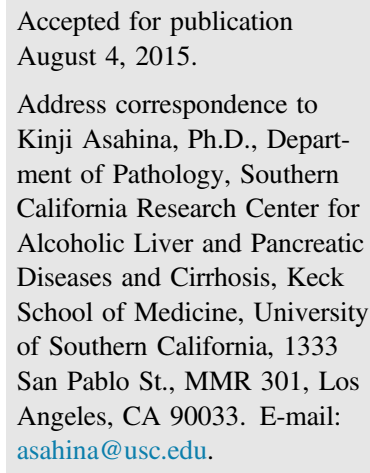

\begin{abstract}
Mesothelial cells (MCs) form a single epithelial layer and line the surface of body cavities and internal organs. Patients who undergo peritoneal dialysis often develop peritoneal fibrosis that is characterized by the accumulation of myofibroblasts in connective tissue. Although MCs are believed to be the source of myofibroblasts, their contribution has remained obscure. We determined the contribution of peritoneal MCs to myofibroblasts in chlorhexidine gluconate (CG)-induced fibrosis compared with that of phenotypic changes of liver MCs. CG injections resulted in disappearance of MCs from the body wall and the accumulation of myofibroblasts in the connective tissue. Conditional linage tracing with Wilms tumor 1 (Wt1)CreERT2 and Rosa26 reporter mice found that $17 \%$ of myofibroblasts were derived from MCs in peritoneal fibrosis. Conditional deletion of transforming growth factor- $\beta$ type II receptor in $W t 1^{+} \mathrm{MCs}$ substantially reduced peritoneal fibrosis. The CG treatment also induced myofibroblastic conversion of MCs in the liver. Lineage tracing with Mesp1-Cre mice revealed that Mesp $1^{+}$mesoderm gave rise to liver MCs but not peritoneal MCs. During recovery from peritoneal fibrosis, peritoneal MCs, but not liver MCs, contribute to the regeneration of the peritoneal mesothelium, indicating an inherent difference between parietal and visceral MCs. In conclusion, MCs partially contribute to myofibroblasts in peritoneal and liver fibrosis, and protection of the MC layer leads to reduced development of fibrous tissue. (Am J Pathol 2015, 185: 3258-3273; http://dx.doi.org/10.1016/j.ajpath.2015.08.009)
\end{abstract}

Mesothelial cells (MCs) are squamous epithelial cells that line the surface of the body cavities (pericardial, pleural, and peritoneal) and internal organs. ${ }^{1}$ By secretion of a lubricating fluid, MCs generate a slippery and antiadhesive surface to facilitate the movement of the internal organs. Although MCs form epithelial sheets with tight junctions and adherence junctions and express epithelial cell markers on the surface of organs, they also express mesenchymal cell markers. In chick embryos, MCs originate from the lateral plate mesoderm. ${ }^{2}$ After gastrulation, the coelomic cavity develops between the lateral plate mesoderm. The dorsal mesoderm associated with the ectoderm gives rise to somatic mesoderm and forms the parietal mesothelium of the body wall. By contrast, the mesoderm developed with the endoderm becomes splanchnic mesoderm and contributes to the visceral mesothelium of the respiratory and digestive tracts. ${ }^{2}$ Similarly, during mouse embryogenesis, MCs in the liver are derived from the mesoderm. ${ }^{3}$ MESP1 is a transcription factor that is transiently expressed in nascent mesoderm during gastrulation and contributes to part of the mesoderm, including the heart. ${ }^{4} \mathrm{~A}$ cell lineage tracing of $\mathrm{Mespl}^{+}$mesoderm revealed its contribution to liver MCs. ${ }^{3,5}$ However, whether both visceral and parietal MCs are derived from the same $\mathrm{Mespl}^{+}$mesoderm in mice remains unknown. In addition, whether MCs in different organs intermingle during development, tissue injury, and regeneration remains elusive.

The multidifferentiation potential of MCs was reported in mouse development. ${ }^{6-11}$ Conditional cell lineage tracing of Wilms tumor $1(W t 1)^{+}$or mesothelin $(M S L N)^{+}$MCs found that MCs migrate inward from the organ surface and give rise to fibroblasts and smooth muscle cells in the developing heart,

Supported by NIH grant R01AA020753 (K.A.) and training program T32HD060549 (I.L.) and the Robert E. and May R. Wright Foundation Award (K.A.).

Disclosures: None declared. 
lung, liver, and intestine. Interestingly, a similar conversion of MCs was recently reported in fibrosis of the adult liver or lung. ${ }^{12,13}$ In the mouse liver, MCs express WT1, cytokeratin 8 (KRT8), and vimentin (VIM) but not E-cadherin (CDH1) and $\alpha$-smooth muscle actin (ACTA2). ${ }^{12}$ On liver injury, $\mathrm{WtI}^{+}$ $\mathrm{MCs}$ give rise to hepatic stellate cells or ACTA2 $2^{+}$myofibroblasts, depending on causes. ${ }^{12}$ Because MCs show an intermediate phenotype between epithelial cells and mesenchymal cells in normal tissues, we defined the change of MCs into myofibroblasts as a mesothelial-mesenchymal transition (MMT) rather than an epithelial-mesenchymal transition. Transforming growth factor (TGF) $\beta$ h has an essential role in the generation of myofibroblasts in organ fibrosis. ${ }^{14} \mathrm{We}$ found that TGF- $\beta$ induces the conversion of MCs into myofibroblasts in vitro. ${ }^{12}$ Furthermore, antagonism of TGF- $\beta$ signaling by treatment with a soluble TGF- $\beta$ type II receptor (STR) led to the suppression of MMT in liver fibrosis. ${ }^{12}$ However, whether direct TGF- $\beta$ signaling is involved in MMT in both visceral and parietal MCs in organ fibrosis remained unclear.

The mesothelium is used as a semipermeable barrier in patients who undergo peritoneal dialysis for end-stage renal disease. ${ }^{15-17}$ Long-term exposure to dialysis solution causes injury to peritoneal MCs and often leads to peritoneal fibrosis and encapsulating peritoneal sclerosis. In the body wall peritoneum, a single layer of MCs is separated from underlying fibroblasts by a basal lamina. Injury to the body wall causes the disappearance of the MC layer from the body wall surface, massive development of connective tissue and blood vessels, and deterioration of the barrier function. ${ }^{15-17}$ Myofibroblasts in the connective tissue actively synthesize extracellular matrices and cause fibrosis in the peritoneum. In patients undergoing peritoneal dialysis, floating MCs are found in the effluents. ${ }^{18}$ These floating MCs were shown to undergo epithelial-mesenchymal transition and to differentiate into fibroblastic cells in culture. ${ }^{18}$ As a result of this finding, the conversion of MCs into mesenchymal cells was extensively studied, and several signaling pathways, such as TGF- $\beta$, IL- $1 \beta$, angiotensin II, and pleiotrophin, were shown to induce this conversion. ${ }^{19-22}$

In addition to MCs, fibroblasts beneath MCs are also suggested to be the source of myofibroblasts in peritoneal fibrosis. ${ }^{23-25}$ Sakai et al ${ }^{24}$ reported that connective tissue growth factor (CTGF) secreted from MCs induces the proliferation and myofibroblastic conversion of fibroblasts in peritoneal fibrosis. Chen et $\mathrm{al}^{25}$ traced liver MCs in mouse peritoneal fibrosis models and indicated that fibroblasts are the main source of myofibroblasts in liver injury. However, the origin of myofibroblasts in peritoneal fibrosis remains to be determined by rigorous cell lineage tracing.

Determination of the origin of myofibroblasts and the mechanism underlying the myofibroblastic conversion of MCs is a prerequisite for the development of antifibrotic drugs. Our aim was to determine the fate of MCs in peritoneal fibrosis by comparing body wall and liver mesothelia as representatives of parietal and visceral organs, respectively. With the use of $W t 1^{\text {CreERT2 }}$ and Rosa26mTmG ${ }^{\text {flox }}\left(R 26 \mathrm{TG}^{\mathrm{fl}}\right)$ mice, we traced peritoneal MCs in the body wall during peritoneal fibrosis and demonstrated the conversion of $\mathrm{WtI}^{+}$ MCs to $\mathrm{ACTA}^{+}$myofibroblasts. We estimated that approximately $17 \%$ of myofibroblasts are derived from MCs. Although MCs are not the main source of myofibroblasts, the suppression of MMT by a conditional deletion of TGF- $\beta$ type II receptor $(T g f b r 2)$ resulted in a substantial reduction in peritoneal fibrosis. We further found that the origin of liver MCs is distinct from that of peritoneal MCs, and liver MCs do not contribute to the regeneration of the peritoneal mesothelium. Our data indicate that protection of the MC layer is beneficial for the suppression of peritoneal fibrosis.

\section{Materials and Methods}

\section{Mice}

Collal $^{\mathrm{GFP}}$ transgenic mice, Mespl ${ }^{\mathrm{Cre}}$, and $\mathrm{Wt}^{\mathrm{CreERT} 2}$ knockin mice were described previously. ${ }^{4,26}$ Rosa26mTmG flox (R26TG $\left.{ }^{\mathrm{fl}} ; 007576\right), T g f b r 2^{\mathrm{fl}}$ (012603), and $U p k 1 b^{\mathrm{RFP}}$ (010984) mice were purchased from The Jackson Laboratory (Bar Harbor, ME). ${ }^{27,28}$ Tamoxifen (Sigma-Aldrich, St. Louis, MO) dissolved in ethanol was emulsified in sesame oil at $12.5 \mathrm{mg} / \mathrm{mL}$ and injected intraperitoneally into the mice (10 to 12 weeks old) at $100 \mu \mathrm{g} / \mathrm{g}$ body weight twice during a 3-day interval. ${ }^{12}$ Two weeks after the last injection, the mice were injected with $0.1 \%$ chlorhexidine gluconate (CG) solution (Sigma-Aldrich) in 15\% ethanol/phosphatebuffered saline at $1.5 \mathrm{~mL} / 100 \mathrm{~g}$ body weight to the right part of the peritoneum every other day. ${ }^{29}$ After 10 injections of $\mathrm{CG}$, the mice developed peritoneal fibrosis. We collected the left part of the peritoneum for histologic analyses to avoid the area damaged by intraperitoneal injections. For the recovery model, the mice that were treated 10 times with CG were maintained for 1 month without treatment. For bromodeoxyuridine (BrdU) incorporation assay, mice were injected with BrdU labeling reagent (Life Technologies, Grand Island, NY; $10 \mu \mathrm{L} / \mathrm{g}$ body weight; $n=3$ ) by intraperitoneal injection 4 hours before sacrifice. For inhibition of TGF- $\beta$ signaling, mice were treated with STR (TGF- $\beta$ R2 Fc chimera; R\&D Systems, Minneapolis, $\mathrm{MN})$ or mouse $\mathrm{IgG} 2 \mathrm{a}$ isotype control $(0.1 \mathrm{mg} / \mathrm{kg}$ body weight; $n=3$ ) with five intraperitoneal injections of $C G$ every other day. Mice were used in accordance with protocols approved by the Institutional Animal Care and Use Committee of the University of Southern California.

\section{Immunohistochemistry, Histology, and Quantification}

Immunohistochemistry was performed as previously described. ${ }^{5,12}$ In brief, the peritoneal body wall or liver tissues were fixed with $4 \%$ paraformaldehyde, incubated with $30 \%$ sucrose in phosphate-buffered saline overnight, and embedded in OCT compound (Sakura Finetech, Torrance, CA). With the use of a cryostat (CM1900; Leica, Buffalo Grove, IL), 7- $\mu \mathrm{m}$ cryosections were cut. After blocking with 5\% goat or donkey 
serum, the sections were incubated with primary antibodies for 1 hour. The primary antibodies for glycoprotein M6a (GPM6A; MBL, Woburn, MA), F4/80, podoplanin (PDPN; eBioscience, San Diego, CA), CD200, CD31 (BD Biosciences, San Jose, CA), VIM (Epitomics, Burlingame, CA), type I collagen (COL I), type IV collagen (COL IV; EMD Millipore, Billerica, MA), green fluorescent protein (GFP; Nacalai Tesque, San Diego, CA; or Life Technologies), desmin (Thermo Scientific, Waltham, MA), and cytokeratin (Dako, Carpinteria, CA) were the same as described previously. ${ }^{5,8,12} \mathrm{We}$ also used rat antiBrdU (200-fold dilution; Abcam, Cambridge, MA; ab6326), rabbit anti-phosphorylated-SMAD2/3 (50-fold dilution; Santa Cruz Biotechnology, Inc., Dallas, TX; sc-11769-R), goat anti-GFP (600-fold dilution; Rockland, Limerick, PA; 600-101-215), or rabbit anti-red fluorescent protein (RFP; 200-fold dilution; Rockland; 600-401-379) antibodies for immunohistochemistry. The primary antibodies were detected with secondary antibodies conjugated with Alexa Fluor dyes (Life Technologies). ACTA2 was detected with anti-actin $\alpha$-smooth muscle-cyanine 3 (200-fold dilution; Sigma-Aldrich; C6198). For immunostaining for KRT8, KRT19 (DSHB, Iowa City, IA), and CDH1 (Life Technologies), samples were fixed with $70 \%$ ethanol for 4 hours as described previously. ${ }^{5,12}$ Tomato fluorescence was bleached with $3 \% \mathrm{H}_{2} \mathrm{O}_{2}$ in methanol for 10 minutes. The sections were counterstained with DAPI (Life Technologies). Fluorescence signals were captured with Nikon 90i microscope and DS-Qi1 digital camera (Nikon, Melville, NY). For histology, sections were stained with hematoxylin and eosin or Sirius red.

To quantify the labeling efficiency of tamoxifen to MCs, we injected tamoxifen twice into $\mathrm{WtI}^{\mathrm{CreERT} /+} ; R 26 \mathrm{TG}^{\mathrm{f} / / \mathrm{ll}}$ mice and sacrificed the mice 2 weeks after the second injection. After immunostaining the body wall and liver with anti-GFP antibodies (three sections for each mouse, three mice in total), digital images were randomly captured with the use of a $20 \times$ objective (60 images for each mouse, 180 images in total), and the length of the $\mathrm{GFP}^{+} \mathrm{MCs}$ was measured with imaging software (NIS-Element BR 3.0; Nikon). To quantify MCderived $\mathrm{ACTA}^{+}$myofibroblasts in the CG model, sections were stained with antibodies against ACTA2 and GFP (three sections for each mouse, three mice in total). After counterstaining with DAPI, images were captured from the connective area, and 18,825 myofibroblasts were counted in the CG model. Similarly, $\mathrm{GFP}^{+}$MCs were counted in the CG reversal model ( $3237 \mathrm{MCs}$ total, $n=3$ mice). In the $T g f b r 2^{\mathrm{f} / \mathrm{fl}}$ model, sections were stained with antibodies against GPM6A, and the length of

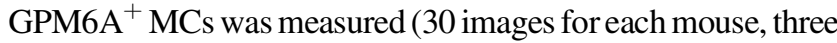
mice in total). To quantify the area of connective tissue in the body wall, at least three sections from each mouse (three mice for each group) were stained with antibodies against ACTA2. After counterstaining with DAPI, at least 20 digital images were randomly captured from a section with the use of a $20 \times$ objective. Each image contained musculature length in the fullscreen mode, and areas of connective tissue were measured by circling the $\mathrm{ACTA} 2^{+}$area between the surface and musculature of the body wall. Similarly, the area of connective tissue, the number of $\mathrm{GFP}^{+} \mathrm{MCs}$, or $\mathrm{BrdU}^{+}$cells were quantified in $T g f b r 2^{\mathrm{f} / / \mathrm{fl}} \mathrm{CG}$ reversal model ( $n=3$ for each model).

\section{qPCR}

Total RNA was extracted with RNAqueous Micro (Life Technologies) and cDNA was synthesized with a SuperScript III kit as previously reported. ${ }^{3}$ Quantitative real-time $\mathrm{PCR}$ (qPCR) was performed with SYBR Green in a ViiA 7 Real-Time PCR System (Life Technologies). The samples were run in triplicate. The relative mRNA levels per samples were calculated by subtracting the detection limit $(40 \mathrm{Ct})$ from the cycle threshold value $(\mathrm{Ct})$ of each gene in the same sample to obtain the $\Delta \mathrm{Ct}$ value. Taking the $\log _{2}$ of $-\Delta C t$ resulted in the relative expression value of each gene for each sample expressed in arbitrary units. Each value was normalized to Gapdh. Primer sequences used are as follows: Ctgf, 5'-ATCCCACCAAAGTGAGAACG-3' (forward) and 5'-ACAGCTGGACTCAGCCTCAT-3' (reverse); Gfp, 5'-CCTACGGCGTGCAGTGCTTCAGC-3' (forward) and 5'-CGGCGAGCTGCACGCTGCCGTCCTC-3' (reverse); Krt19, 5'-CTCGGATTGAGGAGCTGAAC-3' (forward) and $5^{\prime}$-TCACGCTCTGGATCTGTGAC-3' (reverse); Msln, 5'-CTTGGTCGCCTGCTATCTTC-3' (forward) and 5'-ACGGACAGGGCTTTTATCCT-3' (reverse); Upk1b, 5'AGTTGCCTGGTTTGGATTTG- $3^{\prime}$ (forward) and $5^{\prime}$-TGCAGCATCTTGAAAGCCTA-3' (reverse). The primer sequences for Acta2, Cd200, Col1a1, Gapdh, Gpm6a, Krt8, Pdpn, Tgfb1, Tgfb2, Tgfbr2, and Wt1 were the same as described previously.

\section{Isolation and Culture of MCs}

MCs were isolated from the body wall from five mice with the use of magnetic-activated cell sorting (MACS) as previously described. ${ }^{12}$ In brief, the body wall was digested with $1 \mathrm{mg} / \mathrm{mL}$ pronase for 20 minutes. After washing, the cells were incubated with anti-GPM6A antibodies (MBL) and GPM6A $^{+}$MCs were separated with anti-rat microbeads and autoMACS (Miltenyi Biotech, San Diego, CA). The yield of MCs was approximately $1 \times 10^{5} \mathrm{MCs}$ from five mice. MCs $\left(2 \times 10^{4}\right.$ cells $)$ were plated on a collagen-coated 24 -well plate in Dulbecco's modified Eagle's medium with low glucose that contained $10 \%$ fetal bovine serum. The MCs were treated with $10 \mathrm{ng} / \mathrm{mL}$ TGF- $\beta 1$ (Sigma-Aldrich) from day 4 to 8 . MCs were also isolated from five $R 26 T G^{\mathrm{f} / \mathrm{f}} ; T g f b r 2^{\mathrm{f} / \mathrm{fl}}$ mice, treated with an Adenovirus vector carrying LacZ or Cre (multiplicity of infection, 50; Kerafast, Boston, MA) from day 4, and treated with TGF- $\beta 1$ from day 6 for 3 or 12 hours. Cultured MCs were immunostained with antibodies against GFP (500-fold dilution; Nacalai Tesque, Kyoto, Japan), GPM6A (100fold dilution; MBL), ACTA2-cyanine 3 (200-fold dilution; Sigma-Aldrich), and phosphorylated-SMAD3 (100-fold dilution; Abcam; ab52903). The primary antibodies were detected with secondary antibodies conjugated with Alexa 

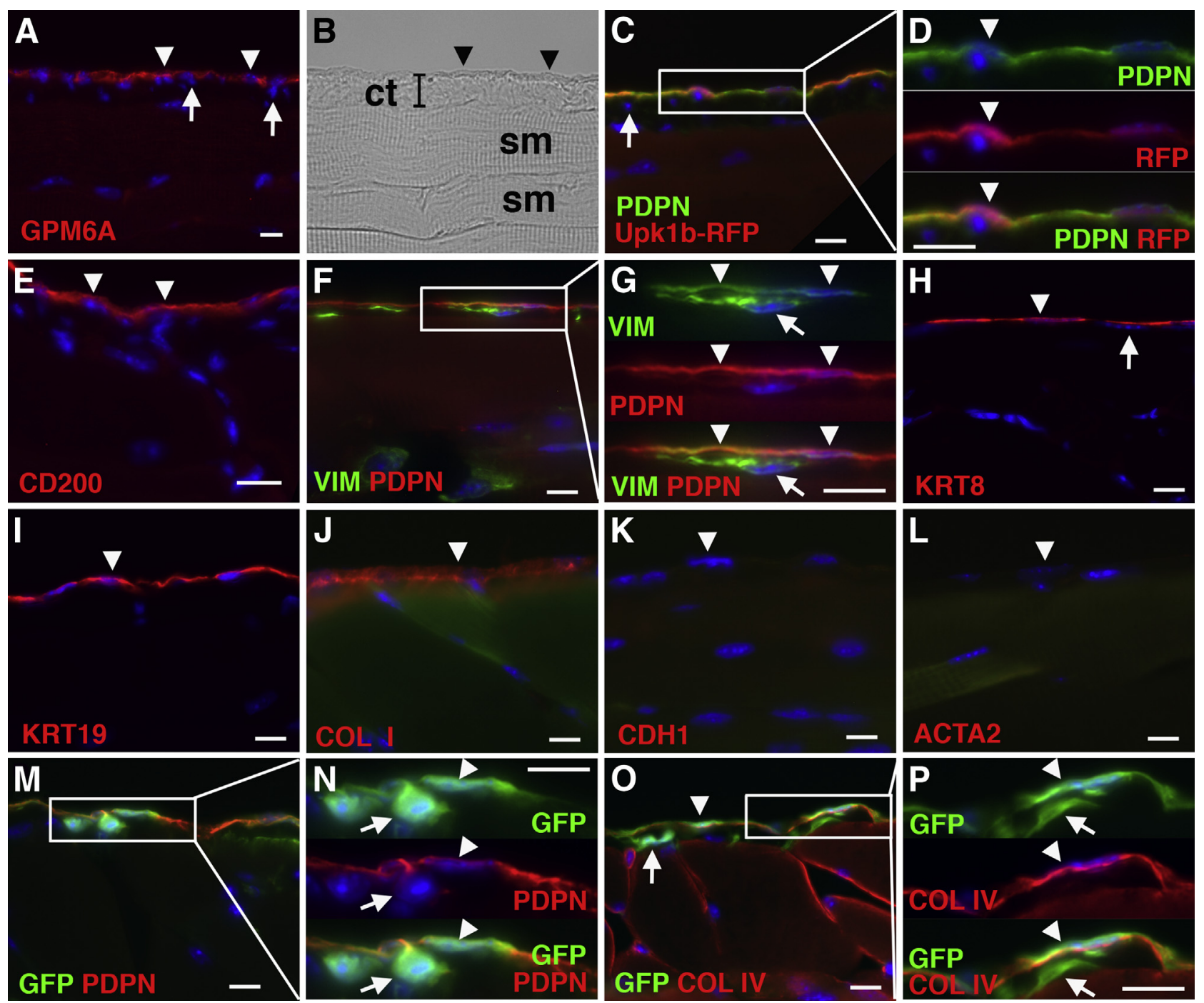

Figure 1 Characterization of peritoneal MCs on the mouse body walls. The body walls from wild-type (A, B, and E-L), Upk1 ${ }^{\mathrm{RFP}}(\mathbf{C}$ and $\mathbf{D})$, and Col1a1 $1^{\mathrm{GFP}}$ (M-P) mice were immunostained with GPM6A (A), PDPN and RFP (C and D), CD200 (E), VIM and PDPN (F and G), KRT8 (H), KRT19 (I), COL I (J), CDH1 (K), ACTA2 (L), GFP and PDPN (M and N), and GFP and COL IV ( $\mathbf{O}$ and $\mathbf{P})$. B: A bright field image of $\mathbf{A}$ is shown. Arrowheads indicate peritoneal MCs on the body wall. Fibroblasts beneath MCs are indicated by arrows. Peritoneal MCs express GPM6A, PDPN, CD200, VIM, KRT8, KRT19, and COL I but not CDH1 and ACTA2 in the normal mouse. The Upk1 $1 b^{\mathrm{RFP}}$ mouse shows specific expression of RFP in PDPN ${ }^{+} \mathrm{MCs}$. MCs and fibroblasts are separated by the basal lamina composed of COL IV, and both cell types express GFP in the Col1 $a^{\text {GFP }}$ mouse. Nuclei were counterstained with DAPI. $n=3$ mice. Scale bars $=10 \mu \mathrm{m}$. ACTA2, $\alpha$-smooth muscle actin; CDH1, E-cadherin; COL I, type I collagen; COL IV, type IV collagen; ct, connective tissue; GFP, green fluorescent protein; GPM6A, glycoprotein M6a; KRT8, cytokeratin 8; KRT19, cytokeratin 19; MC, mesothelial cell; PDPN, podoplanin; RFP, red fluorescent protein; sm, skeletal muscle; VIM, vimentin.

Fluor dyes (Life Technologies). Nuclei were counterstained with DAPI. All experiments were replicated twice.

\section{FACS}

After isolation of MCs from five mice from each genotype by MACS with the use of the anti-GPM6A antibody, the $\mathrm{GPM}^{+} \mathrm{A}^{+} \mathrm{MCs}$ were further incubated with anti-rat IgG Alexa Fluor 647 (Life Technologies). Then, the cells were analyzed with fluorescence-activated cell sorting (FACS) Aria (BD Biosciences) in the University of Southern California Flow Cytometry Core. After excluding cell debris, $\mathrm{GFP}^{+}$Alexa Fluor $647^{+}$and $\mathrm{GFP}^{-}$Alexa Fluor $647^{+} \mathrm{MCs}$ were sorted and were subjected to qPCR. All experiments were replicated twice.

\section{Statistical Analysis}

Statistical significance was assessed by analysis of variance, followed by post hoc Tukey honest significant difference test among multiple samples or Student's $t$-test between two samples. $P<0.05$ was considered statistically significant.

\section{Results}

Phenotype of Peritoneal MCs

We had previously identified Cd200, Gpm6a, Pdpn, and uroplakin $1 \mathrm{~b}$ (Upk1b) as markers for mouse liver MCs. ${ }^{12}$ With the use of immunohistochemistry, we examined 
whether MCs on the body wall similarly express these markers. In this study, we referred to MCs on the body wall as peritoneal MCs and compared their phenotype with that of liver MCs on the liver surface. Similar to liver MCs, peritoneal MCs expressed GPM6A, PDPN, and CD200 on the body wall (Figure 1, A-E). $U p k l b^{\mathrm{RFP}}$ mice expressed RFP exclusively in $\mathrm{PDPN}^{+}$MCs (Figure 1, C and D). No expression of these markers was observed in fibroblasts
A

Wt1 ${ }^{\text {CreERT2/+; }}$

R26TG $^{\text {fl/fl }}$ mouse

Tamoxifen x 2 (Tomato $\rightarrow$ GFP) II $\rightleftarrows \uparrow \uparrow \uparrow \uparrow \uparrow \uparrow \uparrow \uparrow \uparrow \uparrow$ 2wks CG $\times 10$

C
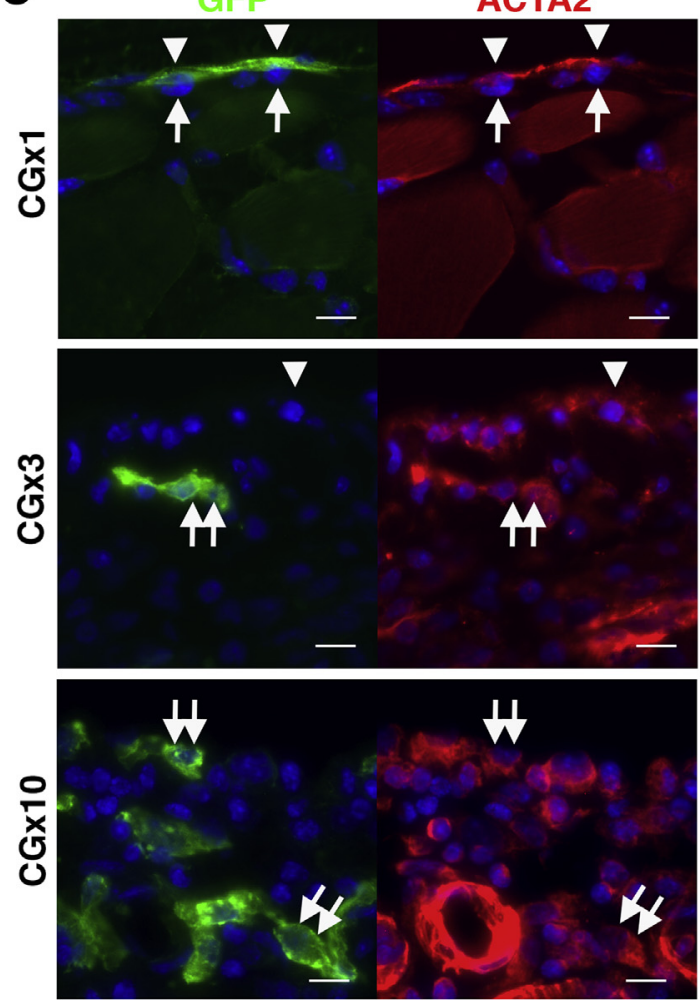

$\mathbf{F}$
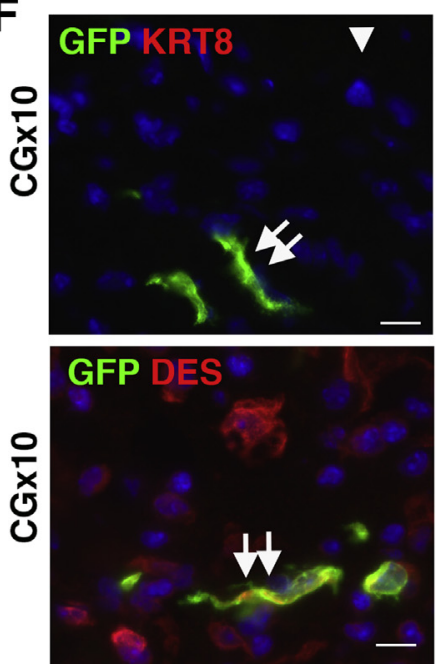

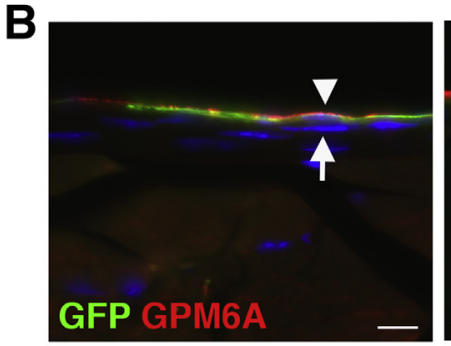

D
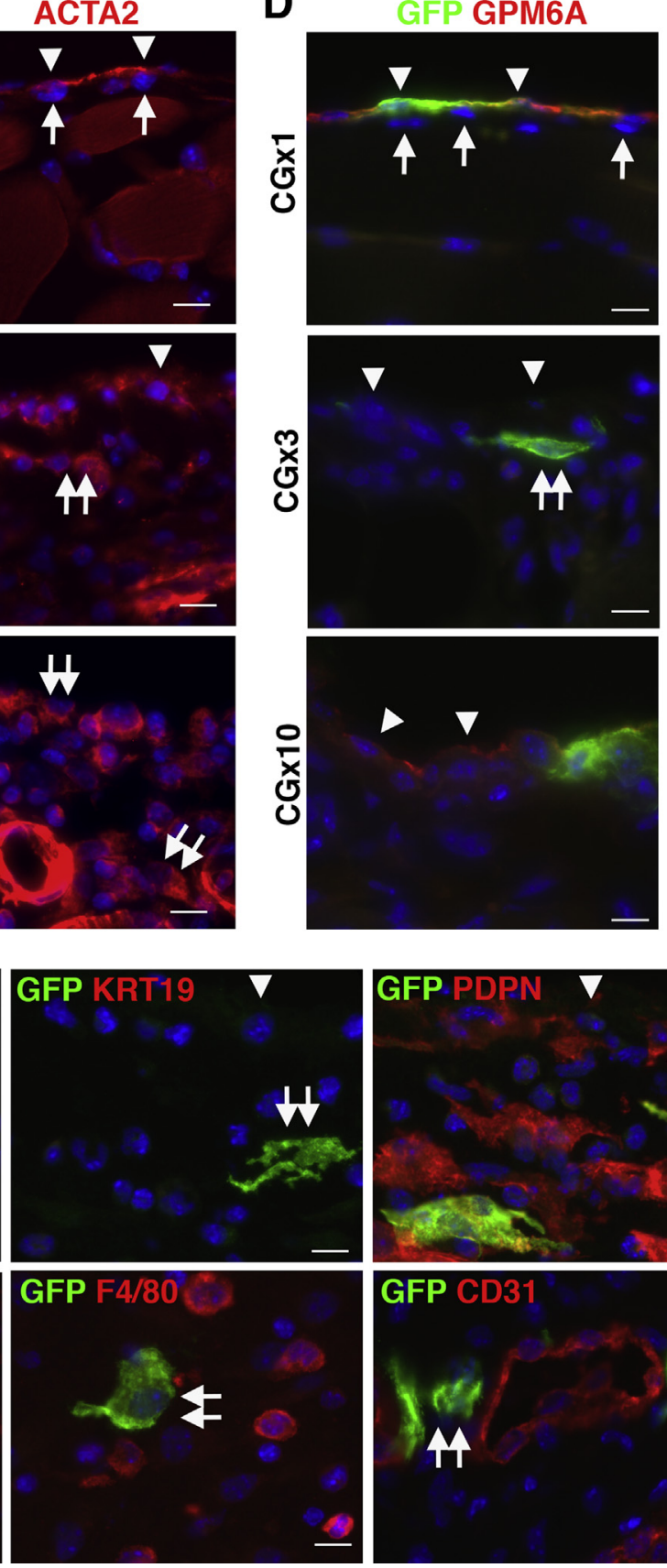
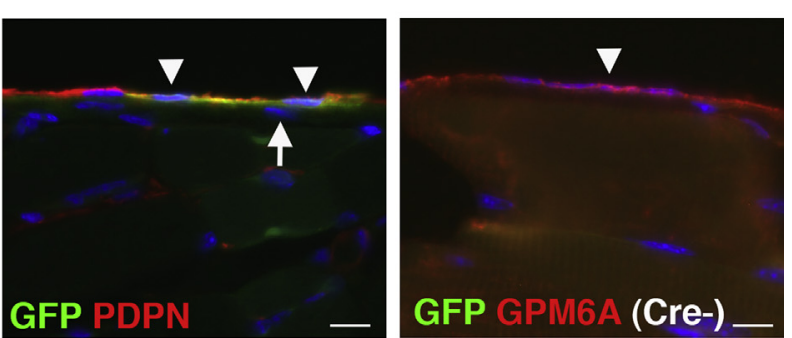

E
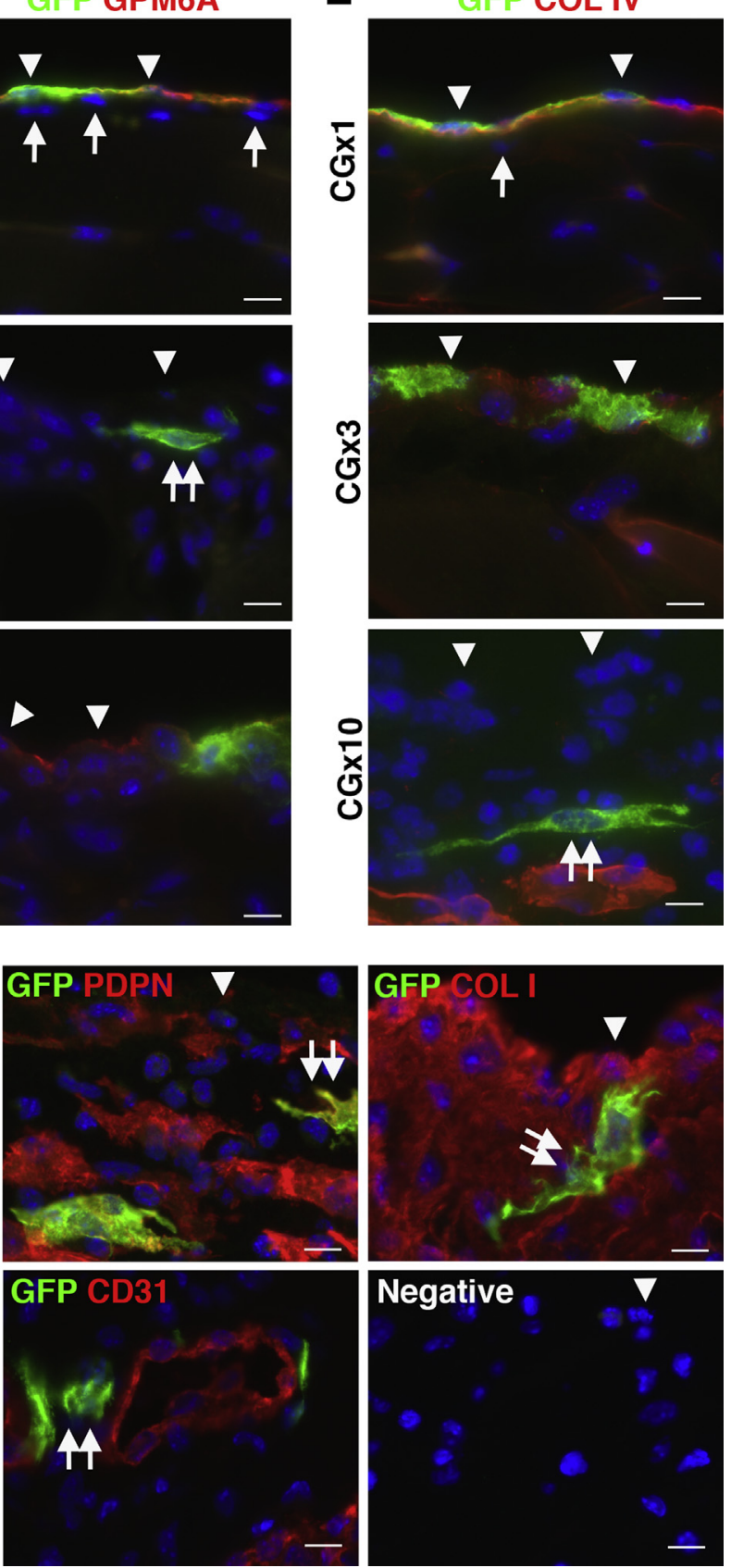
beneath MCs (Figure 1, A and C). MCs expressed both PDPN and VIM, whereas fibroblasts beneath MCs only expressed VIM (Figure 1, F and G). Peritoneal MCs expressed the epithelial markers KRT8 and KRT19 and the mesenchymal markers COL I but not CDH1 and ACTA2 (Figure 1, H-L) in the normal body wall. To test whether peritoneal MCs synthesize Col1a1 mRNA, we analyzed the body wall of a Collal ${ }^{\mathrm{GFP}}$ transgenic mouse. Both PDPN ${ }^{+}$ MCs and PDPN- fibroblasts expressed GFP (Figure 1, M and $\mathrm{N}$ ). Peritoneal MCs and fibroblasts were separated by the basal lamina composed of COL IV in the body wall, and both cell types expressed GFP in normal Collal ${ }^{\text {GFP }}$ mice (Figure 1, $\mathrm{O}$ and $\mathrm{P}$ ). These results indicate that both peritoneal and liver MCs share the same marker expression in normal mice.

\section{Differentiation of MCs into Myofibroblasts in Peritoneal Fibrosis}

To determine whether peritoneal MCs are the source of myofibroblasts in peritoneal fibrosis, we traced the MC lineage with the use of $W t 1^{\mathrm{CreERT} 2}$ and $R 26 \mathrm{TG}^{\mathrm{fl}}$ mice. With tamoxifen treatment, the $\mathrm{WtI}^{\mathrm{CreERT} 2 /+} ; R 26 \mathrm{TG}^{\mathrm{fl} / \mathrm{fl}}$ mouse changes the expression of Tomato into membrane-tagged GFP in $\mathrm{WtI}^{+}$cells by CreERT2 (Figure 2A). For double fluorescent immunohistochemistry, Tomato fluorescence in tissue sections was bleached with $3 \% \mathrm{H}_{2} \mathrm{O}_{2}$ in methanol before staining (Supplemental Figure S1A). Although WT1 expression was not detectable in the peritoneum by immunohistochemistry with available antibodies, tamoxifen injection resulted in specific GFP expression in $12.5 \% \pm 2.1 \%$ of peritoneal MCs that expressed GPM6A or PDPN (Figure 2B). Importantly, no GFP expression was observed in fibroblasts beneath MCs in the peritoneum (Figure 2B). $W_{t 1^{+/+}} ; R 26 \mathrm{TG}^{\mathrm{fl} / \mathrm{fl}}$ control mice did not show GFP signals in MCs after tamoxifen injection (Figure 2B). Two weeks after tamoxifen injection into $\mathrm{Wt}^{\mathrm{CreERT} 2 /+} ; R 26 \mathrm{TG}^{\mathrm{fl} / \mathrm{fl}}$ mice, peritoneal fibrosis was induced by repeated injections of $\mathrm{CG}$, which is known to cause a thickening of the connective tissue. ${ }^{22,29}$ Immunohistochemistry found that $\mathrm{GFP}^{+} \mathrm{MCs}$ begin to express ACTA2 1 day after a single CG injection (Figure 2C). After three CG injections, $\mathrm{GFP}^{+}$cells were observed in the connective tissue, and the cells showed fibroblastic structure and expressed ACTA2 (Figure 2C). After $10 \mathrm{CG}$ injections, ACTA2 ${ }^{+} \mathrm{GFP}^{+}$myofibroblasts were observed in the connective tissue, and $2.1 \% \pm 1.2 \%$ of ACTA2 $^{+}$myofibroblasts expressed GFP (Figure 2C). Considering that all MCs contribute equally to ACTA2 ${ }^{+}$ myofibroblasts and that the labeling efficiency of tamoxifen to MCs is $12.5 \%$, we estimated that $16.8 \%$ of ACTA2 ${ }^{+}$ myofibroblasts are derived from MCs in peritoneal fibrosis.

One day after a single CG injection, $\mathrm{GFP}^{+} \mathrm{MCs}$ still expressed $\mathrm{GPM}^{+} \mathrm{A}^{+}$and were located above the basal lamina that was composed of COL IV (Figure 2, D and E). After three CG injections, the basal lamina began to be disrupted, and the expression of GPM6A decreased on the surface (Figure 2, D and E). After $10 \mathrm{CG}$ injections, a few GPM6A $^{+}$MCs survived, and no expression of COL IV was found on the surface (Figure 2, D and E). In addition, no expression of KRT8 and KRT19 was observed on the surface of the body wall (Figure 2F), indicating the disappearance of the MC layer from the body wall. GFP ${ }^{+}$ myofibroblasts in peritoneal fibrosis expressed PDPN, COL I, and desmin but not KRT8 and KRT19 in the connective tissue (Figure 2F). These $\mathrm{GFP}^{+}$myofibroblasts did not express F4/80, a marker of macrophages, or CD31, a marker of endothelial cells, in the connective tissue (Figure 2F). $W t 1^{\mathrm{CreERT} 2 /+} ; R 26 \mathrm{TG}^{\mathrm{f} / \mathrm{fl}}$ mice without tamoxifen treatment did not show GFP signals in MCs by CG injections (Supplemental Figure S1B), indicating the specific activity of the CreERT2 in MCs. These results demonstrate that MCs undergo MMT and give rise to myofibroblasts in peritoneal fibrosis.

\section{Regeneration of Peritoneal MCs}

We investigated whether the peritoneal mesothelium regenerates if CG treatment is discontinued. We labeled MCs as $\mathrm{GFP}^{+}$cells in $W_{t 1}{ }^{\mathrm{CreERT} 2 /+} ; R 26 \mathrm{TG}^{\mathrm{fl} / \mathrm{fl}}$ mice, induced peritoneal fibrosis with $10 \mathrm{CG}$ injections, and allowed the mice to recover by discontinuing the $\mathrm{CG}$ treatment (Figure 3A). After $10 \mathrm{CG}$ injections, the connective tissue area was significantly increased from $3982 \mu \mathrm{m}^{2}$ to $62,146 \mu \mathrm{m}^{2}$ in sections captured under a $20 \times$ objective, and the area decreased to $18,665 \mu \mathrm{m}^{2}$ at 4 weeks after the last CG injection (Figure 3, B and C), indicating degeneration of the connective tissue. qPCR found

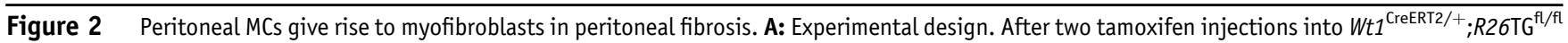
mice, MCs change from the expression of Tomato to membrane-tagged GFP. Two weeks after tamoxifen injection, the mice were treated with CG by 10 intraperitoneal injections. B: Immunohistochemistry of GFP (green) and GPM6A or PDPN (red). After tamoxifen injection into $W t^{{ }^{C r e E R T 2} /+} ;{ }^{2} 26 \mathrm{TG}^{\mathrm{fl} / \mathrm{fl}}$ mice, only ${\mathrm{GPM} 6 \mathrm{~A}^{+} \text {or PDPN }}^{+}$MCs express GFP (arrowheads). Note that no GFP expression in fibroblasts (arrows) is evident. No induction of GFP was observed in the control $W t 1^{+/+} ; R 26 \mathrm{TG} / \mathrm{fl} / \mathrm{fl}$ mice (Cre-) after tamoxifen treatment. C-E: Phenotypic changes of MCs in peritoneal fibrosis. The body wall tissues 1 day after CG injections (1, 3, or 10 times) were immunostained with GFP (green) and ACTA2, GPM6A, or COL IV (red). After a single CG injection, GFP+ MCs begin to express ACTA2 (arrowheads). After three CG injections, $\mathrm{GFP}^{+}$cells in the connective tissue show a myofibroblastic structure with ACTA2 expression but not with GPM6A (double arrows). After 10 CG injections, a few MCs expressing GPM6A survive on the body wall (arrowheads), and no COL IV is observed on the surface of the body wall, indicating denudation of the MC layer. F: Immunohistochemistry of GFP (green) and cell markers (red) after 10 CG injections. Arrowheads indicate the surface of the body wall. MC-derived GFP ${ }^{+}$myofibroblasts express PDPN, COL I, and DES but not KRT8, KRT19, F4/80, and CD31 (double arrows). Immunostaining without primary antibodies does not show signals (Negative). Nuclei were counterstained with DAPI. $n=3$ (B); $n=2$ (C-E, after a single injection and after 3 injections); $n=3$ (C-E, after 10 CG injections); Scale bar $=10 \mu \mathrm{m}$. ACTA2, $\alpha$-smooth muscle actin; CG, chlorhexidine gluconate; COL I, type I collagen; COL IV, type IV collagen; DES, desmin; GFP, green fluorescent protein; GPM6A, glycoprotein M6a; KRT8, cytokeratin 8; KRT19, cytokeratin 19; MC, mesothelial cells; PDPN, podoplanin.
} 


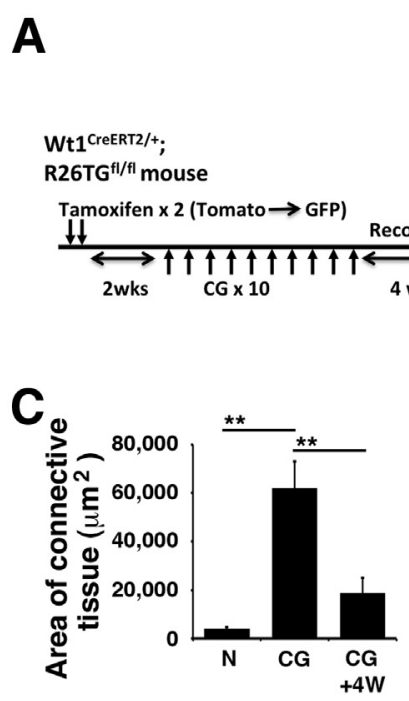

F

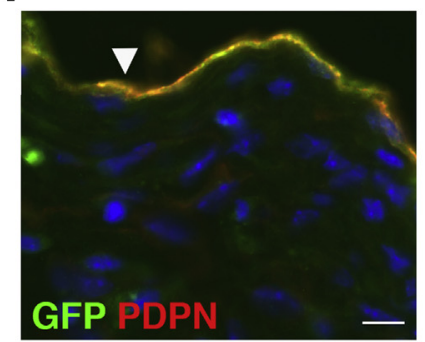

I

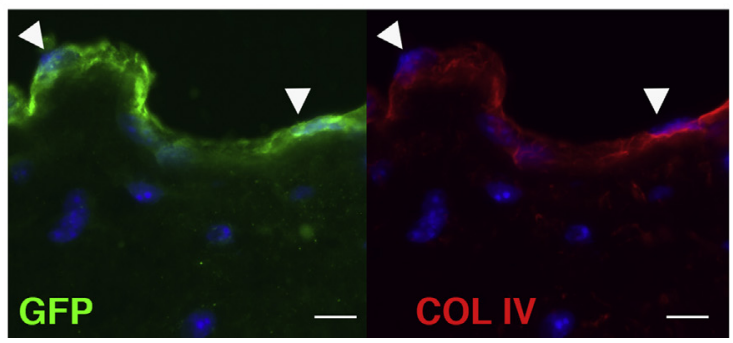

D

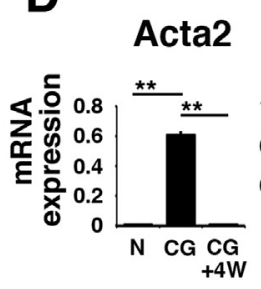

G
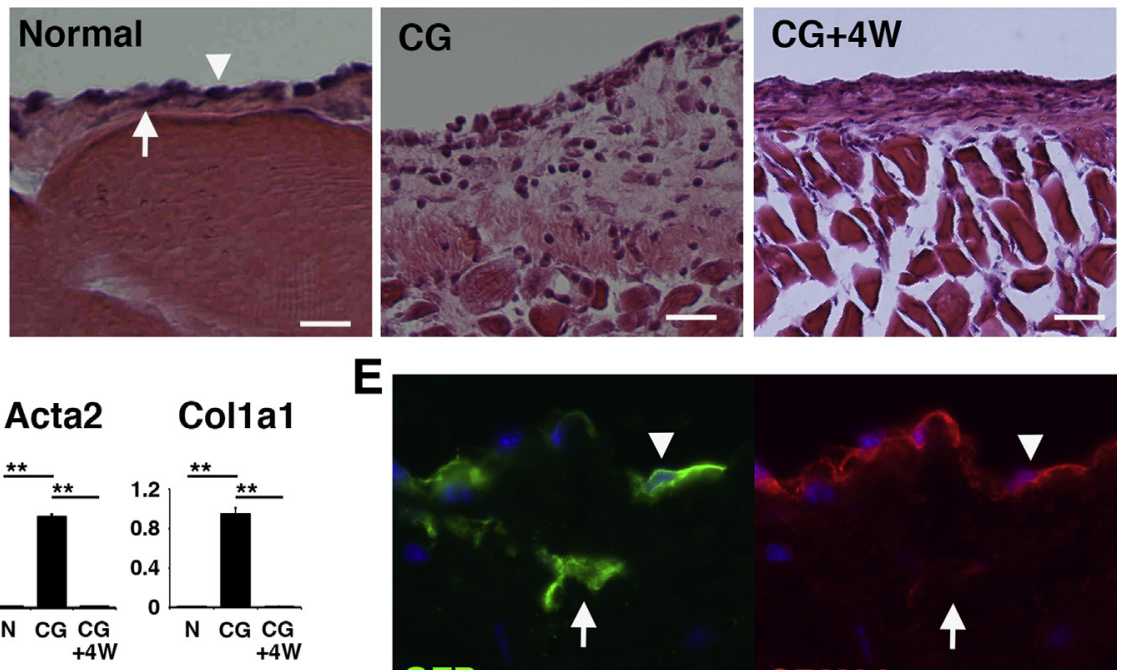

E
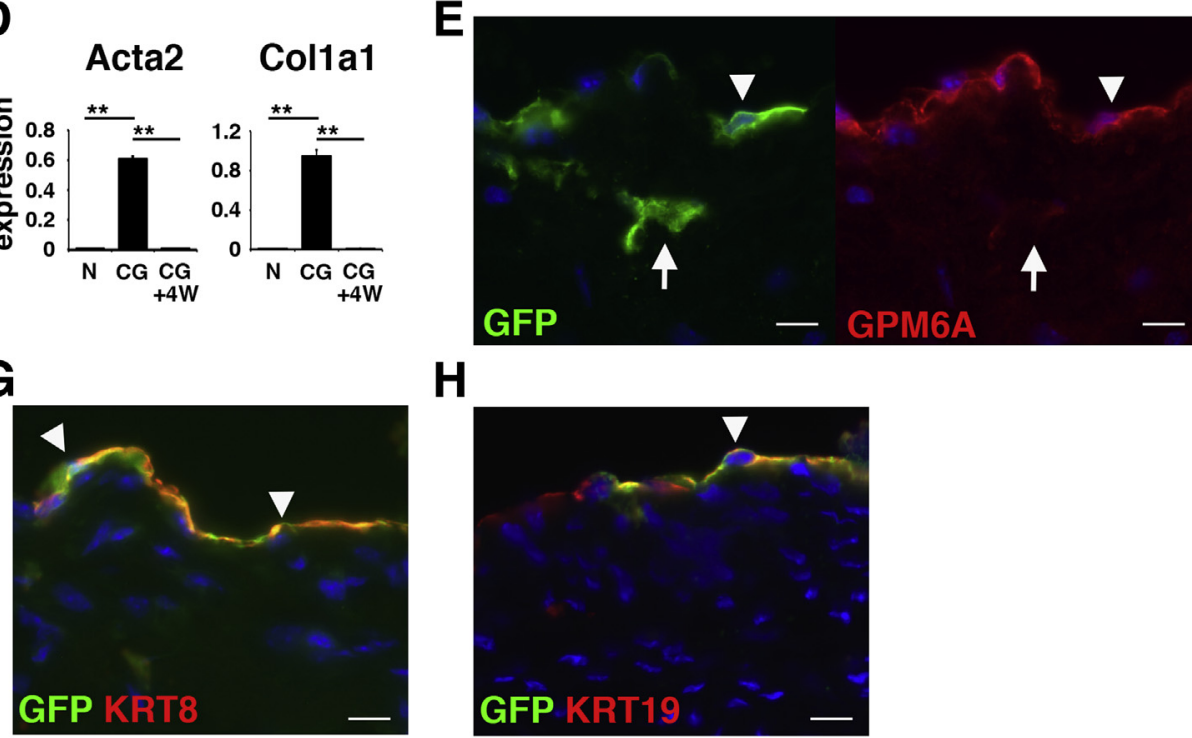

J
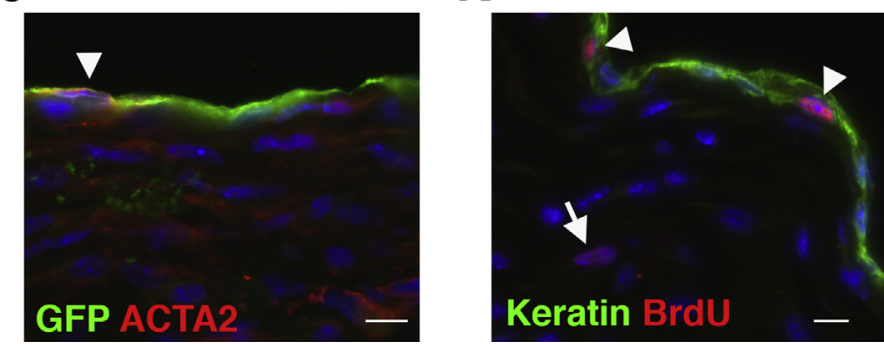

Figure 3 Lineage tracing of MCs during recovery from fibrosis. Regeneration of the MC layer on the body wall during recovery from peritoneal fibrosis. A: By tamoxifen injection into $W t 1^{\text {CreERT } 2 /+} ; R 26 \mathrm{TG}^{\mathrm{fl} / \mathrm{fl}}$ mice, MCs begin to express GFP. Two weeks after tamoxifen injection, peritoneal fibrosis was induced by 10 CG injections, and the mice were allowed to recover for 4 weeks by discontinuing the CG treatment. B: H\&E staining of the body wall before (N), after 10 CG injections (CG), and 4 weeks after the last CG injection (CG+4W). An arrowhead and arrow indicate MCs and fibroblasts, respectively. C: Quantification of the areas of connective tissue in the body wall in B. D: qPCR of mRNA expression in body wall tissues from mice that were N, fibrotic (CG), and recovered 4 weeks after the CG injection (CG+4W). E-J: Immunohistochemistry of the body wall with GFP (green) and GPM6A (E), PDPN (F), KRT8 (G), KRT19 (H), COL IV (I), or ACTA2 (J, red) at 4 weeks after the last CG injection. Arrowheads indicate regenerated peritoneal MCs expressing GFP and markers. Arrows indicate GFP ${ }^{+}$ fibroblasts in the connective tissue. K: BrdU incorporation in the body wall. One week after the last CG injection, the peritoneum was analyzed 4 hours after BrdU treatment. Immunohistochemistry for cytokeratin (Keratin, green) and BrdU (red) shows the incorporation of BrdU in 2.2\% MCs (arrowheads, 910 MCs were counted). An arrow indicates rare myofibroblasts showing nuclear BrdU staining. Nuclei were counterstained with DAPI. $n=3(\mathbf{B}$ and $\mathbf{K}) ;{ }^{* *} P<0.01$. Scale bars: $50 \mu \mathrm{m}(\mathbf{B}) ; 10 \mu \mathrm{m}(\mathbf{E}-\mathbf{K})$. ACTA2, $\alpha$-smooth muscle actin; BrdU, bromodeoxyuridine; CG, chlorhexidine gluconate; COL IV, type IV collagen; GFP, green fluorescent protein; GPM6A, glycoprotein M6a; H\&E, hematoxylin and eosin; KRT8, cytokeratin 8; KRT19, cytokeratin 19; MC, mesothelial cell; N, normal; PDPN, podoplanin; qPCR, quantitative real-time PCR; $4 \mathrm{~W}$, 4 weeks.

increased expression of Acta2 and Colla1 mRNAs by CG injections and that the mRNA expression returned to the baseline level 4 weeks after the last injection (Figure 3D). Immunohistochemistry found that $\mathrm{GPM}^{+} \mathrm{A}^{+} \mathrm{MCs}$ reappeared on the surface of the body wall, and $17.6 \% \pm 4.2 \%$ of MCs co-expressed GFP (Figure 3E), which is similar to the original labeling efficiency of MCs (12.5\%) in the normal body wall
(Figure 2B). Both $\mathrm{GFP}^{+}$and $\mathrm{GFP}^{-} \mathrm{MCs}$ expressed GPM6A, PDPN, KRT8, and KRT19, and COL $\mathrm{IV}^{+}$basal lamina reappeared beneath MCs (Figure 3, E-I). Some of the regenerated MCs still expressed ACTA2 (Figure 3J). $\mathrm{GFP}^{+} \mathrm{ACTA}^{+}$myofibroblasts were rarely observed in the connective tissue (Figure 3J). To test whether survived MCs proliferate and participate in the regeneration of the 
mesothelium, we measured the proliferation by BrdU incorporation assay. One week after the last CG injection, we injected BrdU into mice and collected the peritoneum 4 hours later. Incorporation of $\mathrm{BrdU}$ in nuclei was observed in $2.2 \% \pm 0.9 \%$ of MCs (Figure 3K). Myofibroblasts in the connective tissue rarely showed the BrdU incorporation $(0.2 \% \pm 0.07 \%)$. Few BrdU incorporation was observed in 2 and 4 weeks after the last CG injection (data not shown). These results indicate that the MC layer regenerates and that peritoneal fibrosis is reversible in the mouse model.

\section{Isolation and Culture of Peritoneal MCs}

To examine the mechanisms underlying MMT, we isolated peritoneal MCs from the body wall with the use of antiGPM6A antibodies and MACS. The separated GPM6A ${ }^{+}$ cells expressed mRNAs for MC markers (Gpm6a, Cd200, Msln, Pdpn, Upk1b, Wt1) and epithelial cell markers (Krt8, Krt19) compared with GPM6A ${ }^{-}$cells (Figure 4A), validating the successful purification of peritoneal MCs. In culture, peritoneal MCs formed epithelial colonies up to 7 days (Figure 4B). On day 14, some MCs lost the epithelial cell phenotype and showed a mesenchymal structure. qPCR showed increased expression of Acta2 in cultured MCs (Figure 4C).

TGF- $\beta$ was shown to be a key factor for MMT in the liver. ${ }^{12}$ TGF- $\beta$ binds to a TGF- $\beta$ type II receptor (TGFBR2) dimer, induces phosphorylation of TGF- $\beta$ type I receptor (TGFBR1), and stimulates cells via downstream effectors, including SMAD2 $/ 3{ }^{30}$ In primary peritoneal MCs, TGF- $\beta$ induced a structural change of MCs into myofibroblasts (Figure 4D). According to this change, MCs expressed ACTA2 and increased expression of Acta2 mRNA (Figure 4, $\mathrm{E}$ and $\mathrm{F}$ ). A chemical inhibitor (SB431542) of TGFBR1 blocked the MMT induced by TGF- $\beta$ (Figure 4, D-F). Peritoneal MCs also increased the expression of Ctgf and Pdpn mRNAs in response to TGF- $\beta$ (Figure 4F). Different from liver MCs, ${ }^{12}$ peritoneal MCs did not decrease Gpm6a mRNA in response to TGF- $\beta$ (Figure 4F). TGF- $\beta$ treatment induced the nuclear localization of phosphorylated-SMAD3 in MCs (Figure 4G). These results indicate that TGF- $\beta$ induces MMT in peritoneal MCs.

We confirmed that $\mathrm{WtI}^{+} \mathrm{MCs}$ give rise to $\mathrm{ACTA} 2^{+}$ myofibroblasts in culture. After tamoxifen injection into ${ }_{W t} l^{\mathrm{CreERT} 2 /+} ; R 26 \mathrm{TG}^{\mathrm{f} / \mathrm{fl}}$ mice, peritoneal MCs were isolated by MACS. After 1 week in culture, GPM6A ${ }^{+}$MCs expressed either TOMATO or GFP (Figure $4 \mathrm{H}$ ). After TGF- $\beta$ treatment, the induction of ACTA2 was observed in both $\mathrm{GFP}^{+}$and $\mathrm{GFP}^{-}$myofibroblasts (Figure 4I), indicating that $W t 1^{+}$MCs give rise to $\mathrm{ACTA} 2^{+}$myofibroblasts in culture.

\section{Induction of MMT by TGF- $\beta$}

Next, we examined whether TGF- $\beta$ is responsible for the induction of MMT in peritoneal fibrosis. After labeling MCs as $\mathrm{GFP}^{+}$cells in $W t 1^{\mathrm{CreERT} 2 /+} ; R 26 \mathrm{TG}^{\mathrm{f} / \mathrm{fl}}$ mice, peritoneal fibrosis was induced by $C G$ injections combined with treatment with the use of STR for antagonizing TGF- $\beta$ signaling or control IgG (Figure 5A). Hematoxylin and eosin staining showed that the STR treatment reduced the development of the connective tissue in the body wall compared with the control IgG (Figure 5B). The STR treatment significantly reduced the area of connective tissue from $63,017 \mu \mathrm{m}^{2}$ to $15,657 \mu \mathrm{m}^{2}$ (Figure 5C). Immunohistochemistry showed that the MC layer disappears from the body wall surface and that $\mathrm{ACTA}^{+} \mathrm{GFP}^{+}$myofibroblasts accumulate in the connective tissue in the $\operatorname{IgG}$ group (Figure $5 \mathrm{D}$ ). However, the STR group showed that GPM6A ${ }^{+} \mathrm{GFP}^{+} \mathrm{MCs}$ are present on the surface, and few $\mathrm{GFP}^{+}$myofibroblasts were observed in the connective tissue (Figure 5D). These data indicate that antagonism of TGF- $\beta$ signaling inhibits MMT and peritoneal fibrosis.

\section{Conditional Deletion of Tgfbr2 Gene in MCs}

To further delineate whether direct TGF- $\beta$ signaling in MCs rather than indirect effects of TGF- $\beta$ via other cell types is responsible for MMT, we conditionally deleted exon 4 of the $T g f b r 2$ gene in $\mathrm{WtI}^{+}$MCs. We produced $W t 1^{\mathrm{CreERT} 2 /+} ; R 26 \mathrm{TG}^{\mathrm{f} / \mathrm{fl}} ; \mathrm{Tg} f b r 2^{\mathrm{f} / f \mathrm{ll}}$ mice and conditionally deleted the $T g f b r 2$ gene in $W t 1^{+}$MCs by tamoxifen injection (Figure 5E). CreERT2 abolishes the expression of $T g f b r 2$ while inducing GFP expression. To determine the efficiency of the Tgfbr 2 gene knockout, we injected tamoxifen twice into $W t 1^{\mathrm{CreERT}^{2 /+}} ; R 26 \mathrm{TG}^{\mathrm{fl} / \mathrm{fl}} ; T g f b r 2^{\mathrm{fl} / \mathrm{fl}}$ mice and isolated MCs from the body wall. To efficiently sort MCs by FACS, we first enriched MCs by MACS with the use of an anti-GPM6A antibody anti-rat IgG microbeads. Then, GPM6A ${ }^{+}$MCs were separated into $\mathrm{GFP}^{+} \mathrm{GPM}^{+}$and $\mathrm{GFP}^{-} \mathrm{GPM}^{+} \mathrm{MCs}$ by FACS (Figure 5F). qPCR showed a $77 \%$ reduction of Tgfbr2 mRNA in the $\mathrm{GFP}^{+} \mathrm{MCs}$ compared with $\mathrm{GFP}^{-} \mathrm{MCs}$, whereas increased expression of GFP was observed in the $T g f b r 2^{\mathrm{fl} / \mathrm{fl}}$ conditional knockout mouse (Figure 5G), validating the selective deletion of the Tgfbr2 gene in the $\mathrm{GFP}^{+}$MCs. The deletion of the $T g f b r 2$ gene reduced the expression of Gpm6a mRNA (Figure 5G). The expression of Tgfb1, Tgfb2, and Ctgf mRNAs was not changed between $\mathrm{GFP}^{-}$and $\mathrm{GFP}^{+} \mathrm{MCs}$ by the deletion of the $T g f b r 2$ gene (Figure 5G). After 10 CG injections, the area of connective tissue was significantly decreased in $T g f b r 2^{\mathrm{fl} / \mathrm{fl}}$ mice compared with $T g f b r 2^{\mathrm{f} / /+}$ mice (Figure 5, H and I). $\mathrm{GFP}^{+} \mathrm{ACTA}^{+}$myofibroblasts were observed in the connective tissue, and only $2.1 \% \pm 1.7 \%$ of GPM6A ${ }^{+}$ MCs were found on the body wall surface in the control $T g f b r 2^{\mathrm{fl} /+}$ mice (Figure 5J). However, $T g f b r 2^{\mathrm{ff} / \mathrm{fl}}$ mice showed few $\mathrm{GFP}^{+} \mathrm{ACTA}^{+}$myofibroblasts in the connective tissue, and $20.4 \% \pm 18.7 \%$ of the body wall surface was covered with GPM6A $^{+}$MCs (Figure 5J). We confirmed down-regulation of nuclear phosphorylatedSMAD2/3 in $\mathrm{GFP}^{+}$MCs in $T g f b r 2^{\mathrm{fl} / \mathrm{fl}}$ mice (Figure $5 \mathrm{~K}$ ). 
We isolated MCs from $R 26 T G^{\mathrm{f} / \mathrm{fl}} ; T g f b r 2^{\mathrm{f} / \mathrm{fl}}$ mice and analyzed the effects of the deletion of the Tgfbr2 gene. After treatment with adenovirus carrying Cre, MCs started to express GFP and lost Tgfbr2 mRNA expression (Supplemental Figure S2, A and B). Interestingly, TGF- $\beta 1$ treatment strongly down-regulated the $T g f b r 2$ gene expression in $\mathrm{MCs}$
(Supplemental Figure S2B), suggesting a negative feedback regulation of TGF- $\beta$ signaling. On treatment with TGF- $\beta 1$, MCs infected with adenovirus carrying control LacZ induced nuclear localization of phosphorylated-SMAD3 and the expression of Acta2 and Ctgf mRNAs (Supplemental Figure S2, A and B). In contrast, after Cre expression, GFP ${ }^{+}$

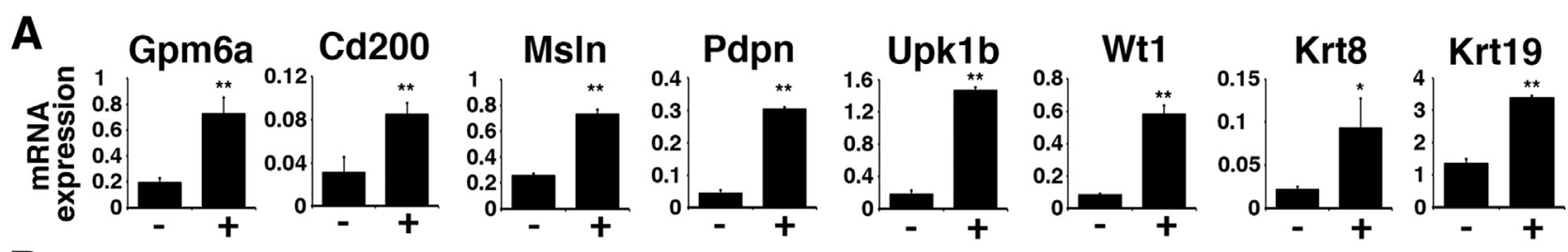

B

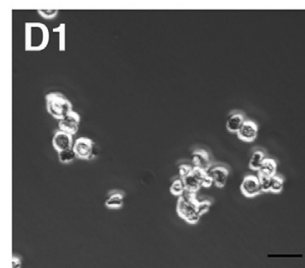

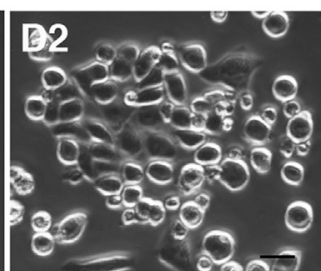

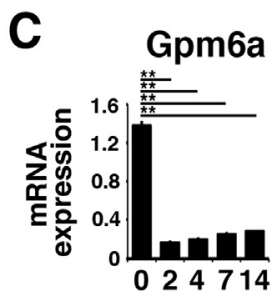

E

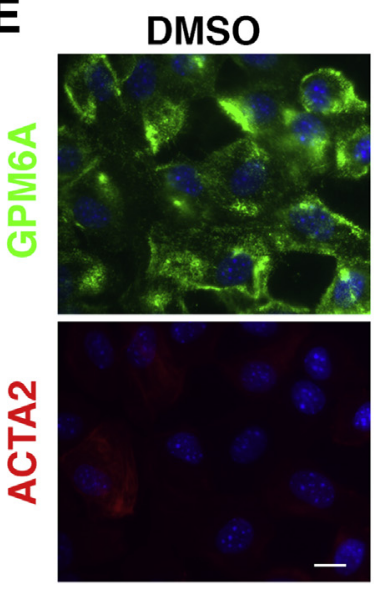

TGF- $\beta$

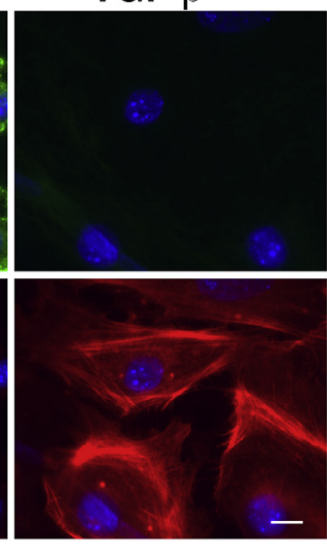

F
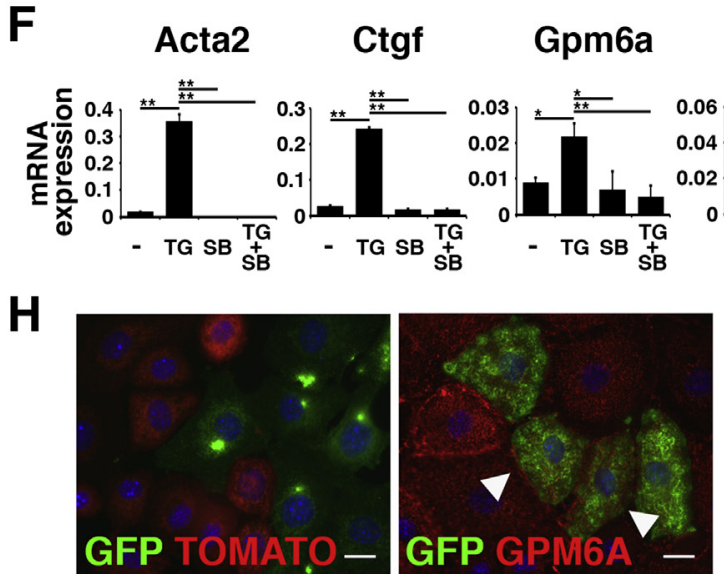
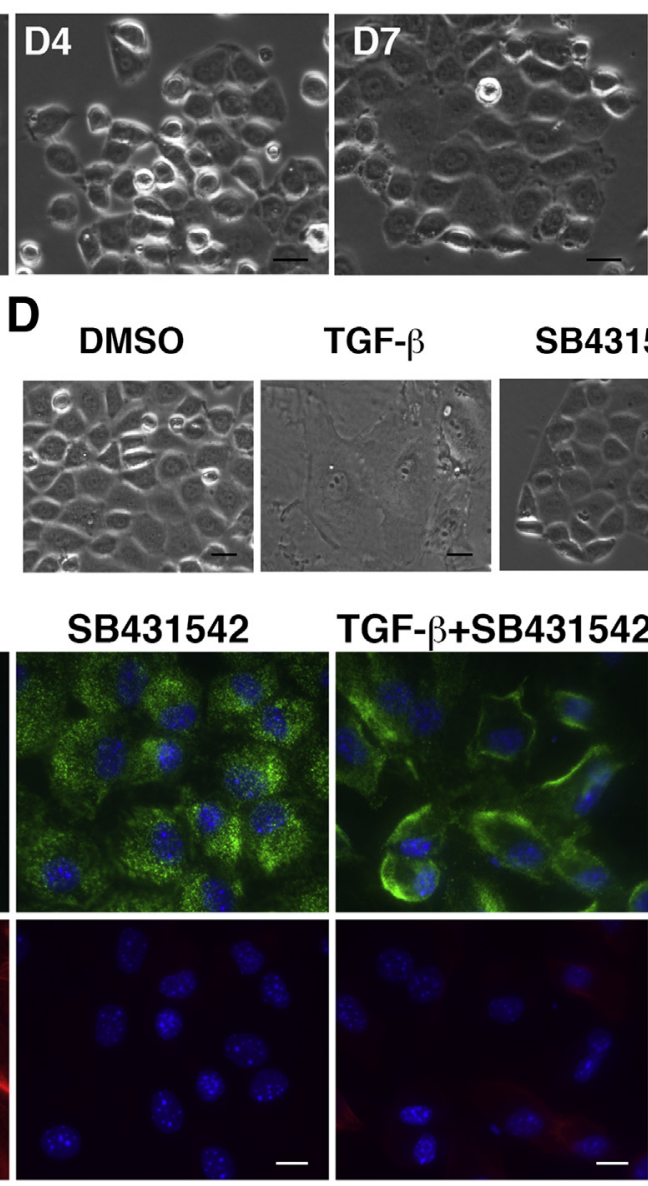

TGF- $\beta+$ SB431542
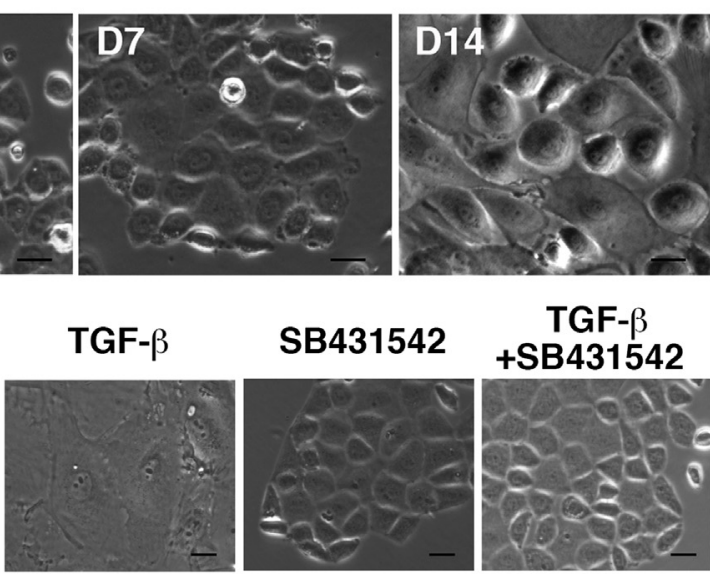

TGF- $\beta$ $+\mathrm{SB} 431542$
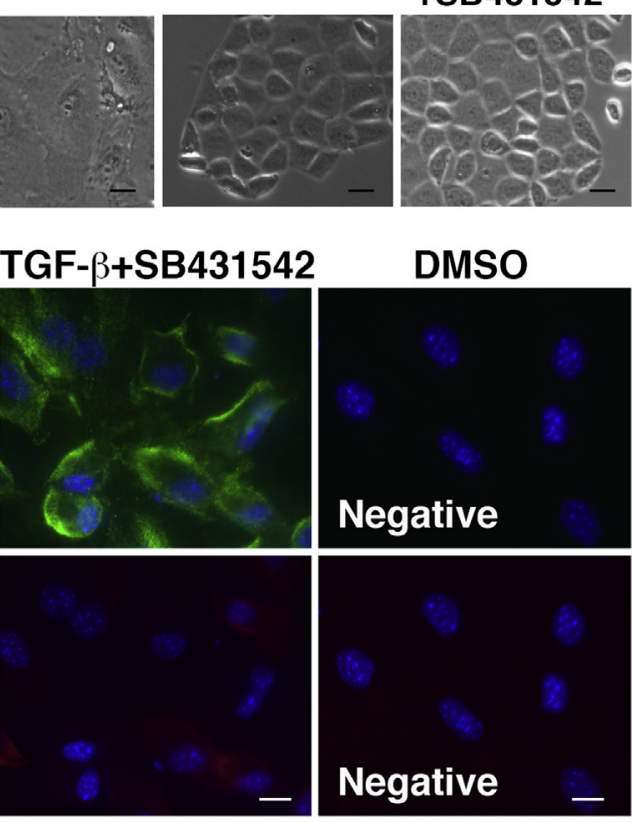

G
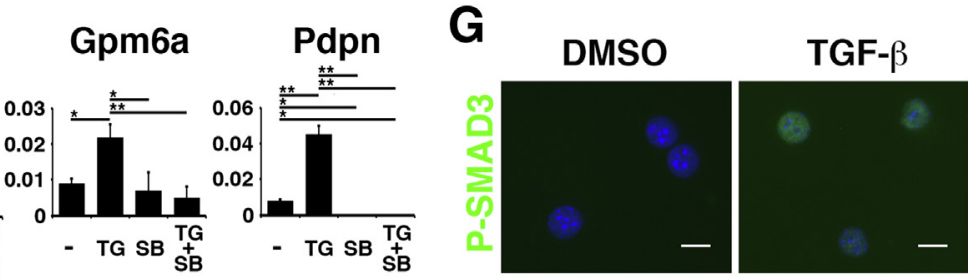

TGF- $\beta$
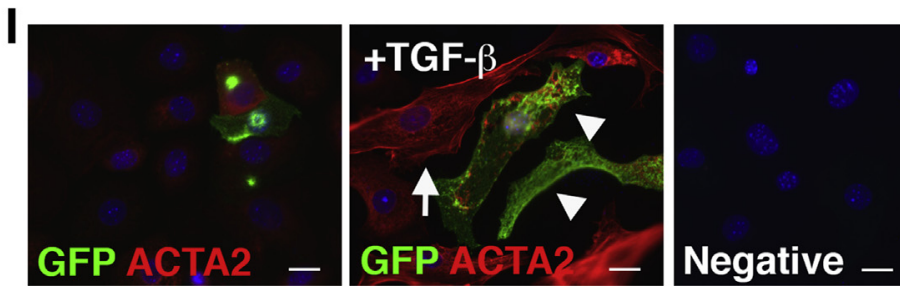
MCs did not show phosphorylated-SMAD3 expression in their nuclei in the presence of TGF- $\beta 1$ and increased expression of Acta2 and Ctgf mRNAs (Supplemental Figure S2, A and B). These data indicate that the canonical TGF- $\beta$ signaling induces the myofibroblastic conversion of peritoneal MCs in vitro.

We also investigated the effects of $T g f b r 2$ gene deletion in MCs during regeneration of the peritoneum from CG-induced peritoneal fibrosis (Supplemental Figure S2C). One month after the last $\mathrm{CG}$ injection, the connective tissue became thinner in both $T g f b r 2^{\mathrm{fl} /+}$ and $T g f b r 2^{\mathrm{fl} / \mathrm{fl}}$ mice similarly (Supplemental Figure S2, D and E) compared with before regeneration (Figure 5, H and I). Immunohistochemistry showed regeneration of $\mathrm{GPM}^{+} \mathrm{A}^{+} \mathrm{MCs}$ on the body wall surface in both genotypes (Supplemental Figure S2F). A few ACTA2 $2^{+}$myofibroblasts were still observed in the $T g f b r 2^{\mathrm{fl} /+}$ but not in the $T g f b r 2^{\mathrm{t} / \mathrm{fl}}$ peritoneum (Supplemental Figure S2G). GFP was observed in $6.8 \% \pm 2.4 \%$ and $2.6 \% \pm 1.3 \%$ of MCs in the $T g f b r 2^{\mathrm{f} / /+}$ and $T g f b r 2^{\mathrm{f} / \mathrm{fl}}$ body walls, respectively. The conditional deletion of $T g f b r 2$ indicated that TGF- $\beta$ signaling is essential for MMT in peritoneal fibrosis.

\section{CG Causes Liver Injury}

CG was used to induce peritoneal fibrosis in the body wall by causing injury to peritoneal MCs. ${ }^{22,29}$ We examined whether $10 \mathrm{CG}$ injections into the peritoneal cavity also causes injury to MCs in the liver as a visceral organ. With the use of the same $W_{t} 1^{\mathrm{CreERT} 2 /+} ; R 26 \mathrm{TG}^{\mathrm{fl} / \mathrm{fl}} ; \mathrm{Tgfbr} 2^{\mathrm{fl} /+}$ or $T g f b r 2^{\mathrm{fl} / \mathrm{fl}}$ mouse and CG treatment, we compared the phenotypes of liver and peritoneal MCs (Figure 6A). By tamoxifen injection, $14.5 \% \pm 6.8 \%$ of GPM6A ${ }^{+}$MCs began to express GFP in the normal liver, and no GFP expression was observed except in MCs (Figure 6B). MCs did not express ACTA2 in the normal liver (Figure 6B). Sirius red staining revealed that normal mouse liver has thin collagen lamella beneath MCs, and its thickness is approximately $2.4 \pm 0.9 \mu \mathrm{m}$ (Figure 6, C and D). After CG injections, the thickness of the collagen lamella was increased to $10.2 \pm 1.5 \mu \mathrm{m}$ beneath the liver surface in control $\mathrm{Tgfbr}^{+/+}$mice (Figure 6, C and D). The increase of collagen lamella by $\mathrm{CG}$ treatment was reduced to $4.8 \pm 0.8 \mu \mathrm{m}$ in $T g f b r 2^{\mathrm{f} / / \mathrm{fl}}$ mice but not in $T g f b r 2^{\mathrm{fl} /+}$ mice (Figure 6, C and D). Immunohistochemistry showed that $\mathrm{CG}$ treatment induced accumulation of $\mathrm{ACTA}^{+}$myofibroblasts only beneath the liver surface in $T g f b r 2^{\mathrm{f} / /+}$ mice and $11.9 \% \pm 9.2 \%$ of ACTA2 ${ }^{+}$ myofibroblasts co-expressed GFP (Figure 6E). Compared with $T g f b r 2^{\mathrm{f} / /+}$ mice, $T g f b r 2^{\mathrm{fl} / \mathrm{fl}}$ mice showed less accumulation of $\mathrm{ACTA}^{+}$myofibroblasts beneath MCs, and a few $\mathrm{GFP}^{+}$ $\mathrm{ACTA}^{+}$myofibroblasts were observed (Figure $6 \mathrm{E}$ ). These data indicate that $\mathrm{CG}$ induces the myofibroblastic conversion of liver MCs and that TGF- $\beta$ signaling is responsible for MMT. Different from the peritoneum, GPM6A ${ }^{+}$MCs remained on the liver surface without expression of ACTA2 after CG injections (Figure 6, E and F). On differentiation to $\mathrm{ACTA}^{+}$myofibroblasts, MCs lost the expression of GPM6A, an MC marker, and $\mathrm{GFP}^{+}$MC-derived myofibroblasts were observed up to $55 \mu \mathrm{m}$ in depth from the surface (Figure 6, E and F). Nuclear localization of phosphorylated-SMAD2/3 was broadly observed in MCs, myofibroblasts, and hepatocytes in the liver by $\mathrm{CG}$ treatment (Figure 6G). Its expression was down-regulated in $\mathrm{GFP}^{+}$cells in $T g f b r 2^{\mathrm{f} / / \mathrm{l}}$ mice compared with $T g f b r 2^{\mathrm{fl} /+}$ mice (Figure 6G). One month after the last CG injection, the thickness of the collagen lamella was reduced to 4 to $5.5 \mu \mathrm{m}$ in all genotypes (Figure 6, C and D). Immunohistochemistry showed the presence of $\mathrm{GFP}^{+} \mathrm{MCs}$ on the liver surface without ACTA2 expression after regeneration (Figure $6 \mathrm{H}$ ).

\section{Distinct Origins of Peritoneal and Liver MCs}

In contrast to liver MCs, peritoneal MCs begin to express ACTA2 on CG treatment, and the MC layer largely disappears in peritoneal fibrosis (Figure 2, C and D). During regeneration, the surviving peritoneal MCs might be the source of regeneration of the peritoneal mesothelium. In addition to this possibility, visceral MCs might traverse the peritoneal cavity, integrate into the parietal mesothelium, and participate in regeneration of the peritoneal $\mathrm{MC}$ layer during regeneration. In fact, floating MCs were isolated from the peritoneal cavity in patients who underwent peritoneal dialysis. ${ }^{18}$ To test whether liver MCs traverse the peritoneal cavity and form a peritoneal MC layer in CG-induced fibrosis, we analyzed the developmental lineages of liver and peritoneal MCs. We previously reported that liver MCs are derived from Mesp $1^{+}$ mesoderm. ${ }^{3,5}$ MESP1 is a transcription factor that is transiently expressed in nascent mesoderm during gastrulation and

\footnotetext{
Figure 4 Induction of peritoneal MMT by TGF- $\beta$ in vitro. Peritoneal MCs were isolated from the mouse body wall with the use of anti-GPM6A antibodies and MACS. A: qPCR of GPM6A ${ }^{-}(-)$and GPM6A ${ }^{+}$cells $(+)$separated from body wall cells by MACS. The GPM6A ${ }^{+}$fraction highly expresses mRNAs for Gpm6a, Cd200, Msln, Pdpn, Upk1b, Wt1, Krt8, and Krt19. B: Culture of GPM6A ${ }^{+}$peritoneal MCs. MCs form epithelial colonies. Some MCs lose epithelial cell structure in culture. C: $q P C R$ of peritoneal MCs in culture. MCs increase the expression of Acta2 mRNA in accordance with the structural changes shown in B. D: Peritoneal MCs were cultured in the presence or absence of TGF- $\beta$ and SB431542. TGF- $\beta$ induces MMT, whereas SB431542 blocks TGF- $\beta$-induced structural changes. E: Immunocytochemistry of cultured peritoneal MCs with antibodies against ACTA2 (red) and GPM6A (green). TGF- $\beta$ strongly induces the expression of ACTA2, whereas SB431542 blocks the induction of ACTA2. F: qPCR of cultured peritoneal MCs in the presence of DMSO (-), TG, SB, and both TG and SB. TGF- $\beta$ induces the expression of Acta2, Ctgf, Gpm6a, and Pdpn mRNAs. G: Immunocytochemistry of cultured peritoneal MCs. TGF- $\beta$ induces the nuclear localization of P-SMAD3 (green). $\mathbf{H}$ and I: Lineage tracing of Wt1 ${ }^{+}$peritoneal MCs in culture. After tamoxifen injection to Wt $1^{\text {CreeRT2/+}} ; R 26 \mathrm{TG} \mathrm{fl}^{\mathrm{fl} / \mathrm{fl}}$ mice, GPM6A ${ }^{+}$MCs were isolated by MACS. H: MCs express either Tomato (red) or GFP (green). Arrowheads indicate MCs co-expressing GFP (green) and GPM6A (red). I: With TGF- $\beta$ treatment $(+\mathrm{TGF}-\beta)$, both $\mathrm{GFP}^{+}$(arrowheads) and GFP- MC-derived myofibroblasts (arrow) express ACTA2. No signals were detected in a negative control without primary antibodies (negative). Nuclei were counterstained with DAPI. ${ }^{*} P<0.05,{ }^{*} * P<0.01$. Scale bars: $20 \mu \mathrm{m}(\mathbf{B}$ and $\mathbf{D}) ; 10 \mu \mathrm{m}$ (E, and G-I). ACTA2, $\alpha$-smooth muscle actin; DMSO, dimethyl sulfoxide; GFP, Green fluorescent protein; GPM6A, glycoprotein M6a; MACS, magnetically activated cell sorting; MC, mesothelial cell; MMT, mesothelial-mesenchymal transition; P-SMAD3, phosphorylated-SMAD3; qPCR, quantitative real-time PCR; SB, presence of SB431542; SB431542, inhibitor for TGFBR1; TG, presence of TGF- $\beta$; TGF, transforming growth factor.
} 
A

Wt1 ${ }^{\text {CreERT2/+. }}$

R26TG $^{\text {fl/fil }}$ mouse

Tamoxifen $\times 2$ (Tomato $\rightarrow$ GFP)

Tam

$\longleftrightarrow \uparrow \uparrow \uparrow \uparrow$

2wks IgG or STR with CG $\times 5$

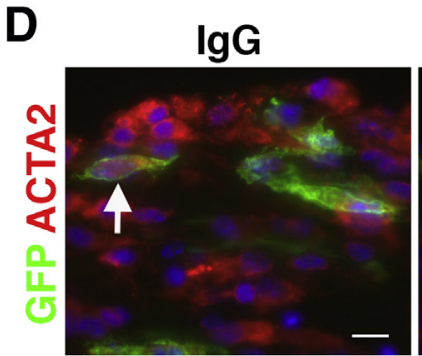

E

Wt1 ${ }^{\text {CreERT2/+; }} ;$ R26TG $^{\text {fl/f }}$

; Tgfbr2 $2^{\mathrm{n} / \mathrm{f}}$ mouse

Tamoxifen $\times 2$ (Tomato It

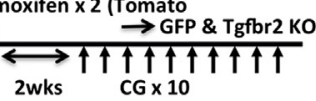

$\mathbf{F}$$$
\text { cexio }
$$

B

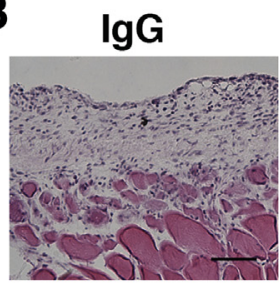

STR

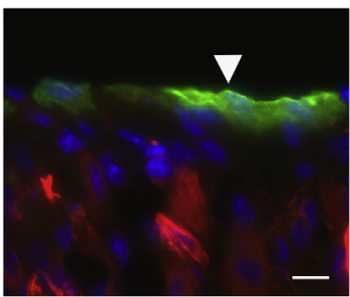

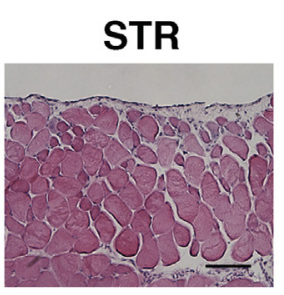

$\lg G$

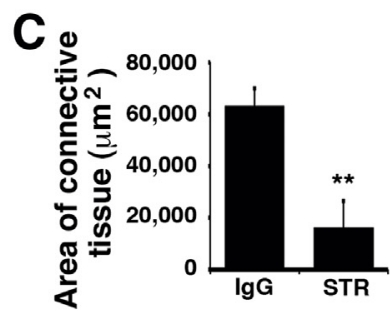

STR
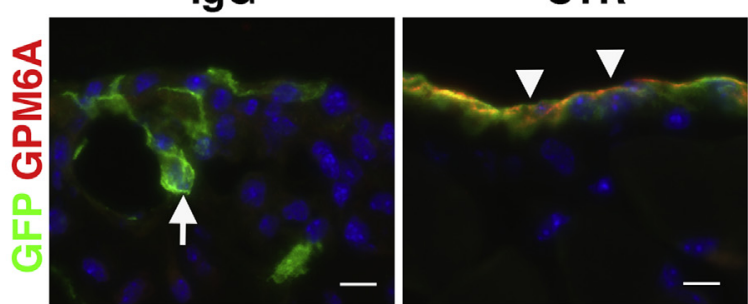

$\longrightarrow$

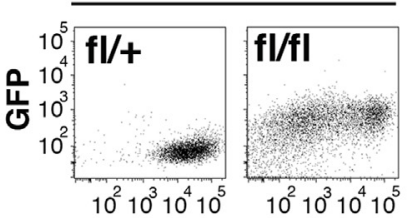

GPM6A

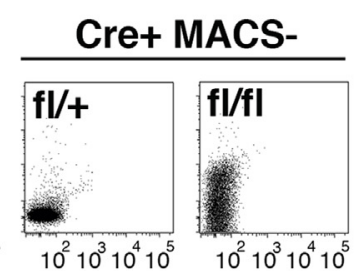

GPM6A

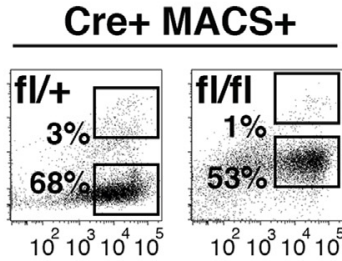

GPM6A

G
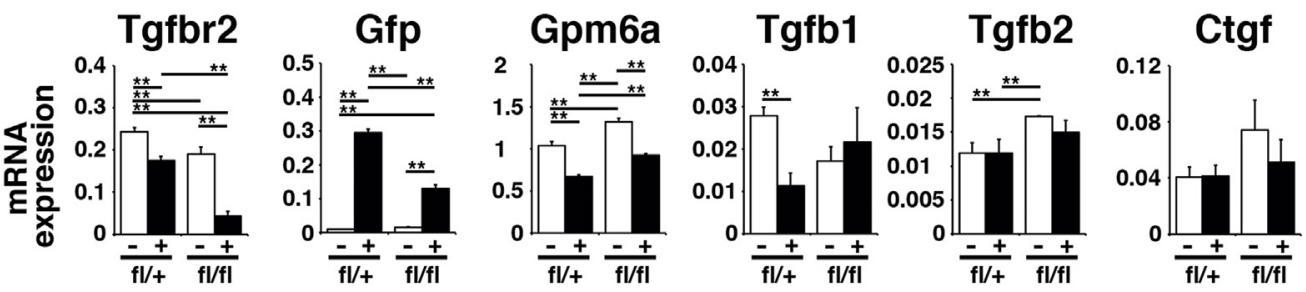

H

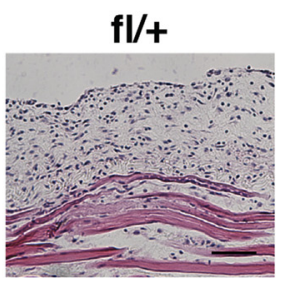

J

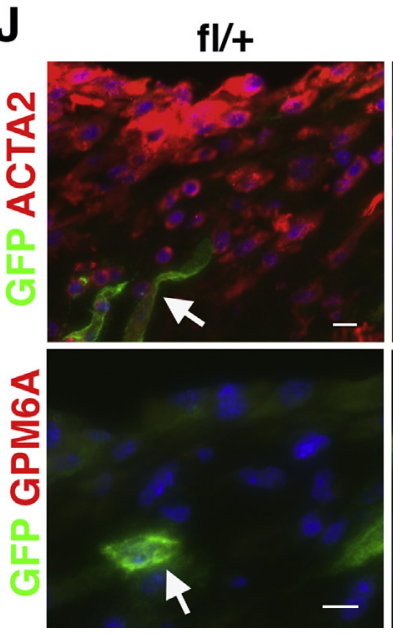

$\mathrm{fl} / \mathrm{fl}$

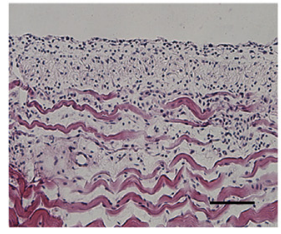

fl/fl

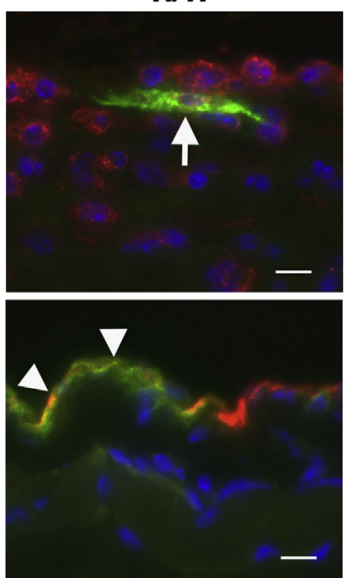

I

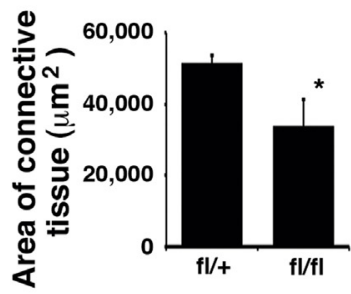

K

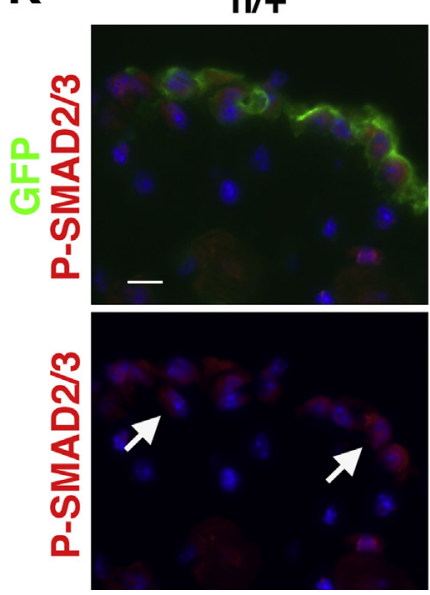

fl/fl

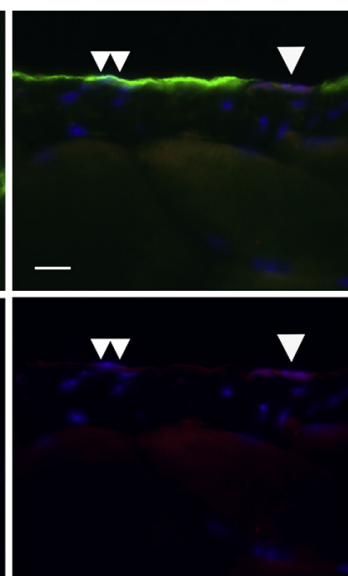


contributes to a part of the mesoderm. ${ }^{4}$ With the use of $M e s p 1^{\mathrm{Cre} /+} ; R 26 \mathrm{TG}^{\mathrm{f} / \mathrm{fl}}$ mice, we evaluated whether both liver and peritoneal MCs are derived from the same mesodermal origin (Figure 6I). In the normal Mesp ${ }^{\mathrm{Cre} /+} ; R 26 \mathrm{TG}^{\mathrm{fl} / \mathrm{ll}}$ liver, $48.8 \% \pm 11.7 \%$ of MCs express GFP (Figure $6 \mathrm{~J}$ ). By contrast, no $\mathrm{GFP}^{+}$MCs were observed in the body wall (Figure $6 \mathrm{~J}$ ). Hepatic stellate cells in the liver and fibroblasts in the peritoneum expressed GFP (Figure $6 \mathrm{~J}$ ). The cell lineage tracing indicates that the origin of peritoneal MCs is distinct from that of liver MCs.

Next, we analyzed whether liver MCs contribute to the regeneration of the peritoneal MC layer. We induced peritoneal fibrosis with the use of $10 \mathrm{CG}$ injections into the $M e s p 1^{\mathrm{Cre} /+} ; R 26 \mathrm{TG}^{\mathrm{fl} / \mathrm{fl}}$ mice and analyzed the body wall 4 weeks after the last CG injection (Figure 6I). After recovery from CG-induced injury, GFP expression was observed in fibroblasts in the connective tissue (Figure 6K), but it was rare in regenerated MCs in the body wall (Figure 6K), indicating a negligible contribution of liver MCs to the regeneration of the peritoneal mesothelium during the recovery from peritoneal fibrosis.

\section{Discussion}

The transition of MCs to myofibroblasts was suggested in peritoneal fibrosis. However, no lineage tracing study with the use of mouse genetics was conducted to demonstrate this hypothesis. With the use of conditional cell lineage tracing, this study reports for the first time that peritoneal MCs give rise to myofibroblasts in a mouse peritoneal fibrosis model. On the basis of the labeling efficiency of MCs in the $W_{t 1}{ }^{\mathrm{CreERT} 2 /+} ; R 26 \mathrm{TG}^{\mathrm{fl} / \mathrm{fl}}$ mouse, we estimated that $16.8 \%$ of myofibroblasts are derived from peritoneal MCs. What are other sources of myofibroblasts during peritoneal fibrosis? Fibroblasts that are positive for VIM exist between MCs and skeletal muscles in the peritoneum and are likely to be another source of myofibroblasts. Although we attempted to trace lineages of fibroblasts with the use of Collal ${ }^{\text {CreERT2 }}$ mice (The Jackson Laboratory; catalog no. 016241), we failed to label fibroblasts by tamoxifen injections (data not shown). More CreERT2 mouse lines will be necessary to quantify the contribution of fibroblasts to myofibroblasts in peritoneal fibrosis. Nevertheless, the present study indicates that MCs partially contribute to myofibroblasts in peritoneal and liver fibrosis.

With the use of peritoneal fibrosis induced by the injection of hypochlorite, dialysis solution, or TGF- $\beta$ in the same $W_{t 1}{ }^{\text {CreERT2 }}$ or Collal ${ }^{\mathrm{GFP}}$ mice, Chen et $\mathrm{al}^{25}$ reported that the contribution of MCs to myofibroblasts is rare in the liver. By treating $\mathrm{Wt}^{\mathrm{CreERT2}}$ mice with tamoxifen 10 times through oral gavage, the investigators labeled $83 \%$ of MCs and $24 \%$ of fibroblasts beneath MCs in the liver and concluded that fibroblasts beneath MCs are the main source of myofibroblasts in the liver. In addition, they reported that MCs are negative for GFP in the Collal ${ }^{\mathrm{GFP}}$ mouse liver. Chen et $\mathrm{al}^{25}$ did not analyze the body wall in these models. In our experimental condition, we labeled $12.5 \%$ and $14.5 \%$ of MCs in the body wall and liver, respectively, in the $\mathrm{Wt}^{\mathrm{CreERT2}}$ mice, and we did not observe any labeling in fibroblasts in these tissues. Different from their report, we found that the normal Collal ${ }^{\text {GFP }}$ mouse expresses GFP in both MCs and fibroblasts in the normal body wall and liver. These differences may cause different outcomes between the present study and their study.

TGF- $\beta$ was shown to be a main inducer of MMT in peritoneal fibrosis. ${ }^{19,20,31}$ Similar to these reports, we found that TGF- $\beta$ induces MMT in peritoneal MCs. Suppression of TGF$\beta$ signaling with STR substantially reduced the number of MC-derived myofibroblasts and the area of the connective tissue induced by CG injections. Furthermore, the MC layer was protected by STR treatment in the CG model. TGF- $\beta$ is known to induce fibrosis in organs by activating myofibroblasts, whereas it suppresses inflammation and carcinogenesis in epithelial cells. ${ }^{30}$ In fact, the overexpression of a STR plasmid enhanced inflammation and failed to attenuate

\footnotetext{
Figure 5 Essential role of TGF- $\beta$ signaling in peritoneal MMT in fibrosis. TGF- $\beta$ signaling was inhibited with the use of STR (A-D) or conditional deletion of the Tgfbr2 gene in peritoneal MCS (E-K). A: Experimental design. After labeling peritoneal MCs as GFP ${ }^{+}$cells by tamoxifen injection in $W t 1^{\text {CreeRT2/+ }} ; R 26 \mathrm{TG}^{\mathrm{fl} / \mathrm{fl}}$ mice, peritoneal fibrosis was induced by CG injections with either STR or IgG injection five times. B: H\&E staining of the body wall treated with an IgG control or STR in CGinduced fibrosis. C: Quantification of the area of the connective tissue treated with an IgG control or STR in B. D: Immunohistochemistry of the body wall with the use of anti-GFP antibodies (green) and ACTA2 or GPM6A antibodies (red). Arrowheads and arrows indicate MCs and myofibroblasts, respectively. The STR treatment inhibits MMT on the body wall. E: Experimental design of the conditional deletion of the Tgfbr2 gene. The tamoxifen-induced CreERT2 in peritoneal MCs activates the expression of GFP while deleting the Tgfbr2 gene. F: FACS of MCs isolated from the $\mathrm{fl} /+$ or $\mathrm{fl} / \mathrm{fl}$ mouse. After isolation of MCs with the use of the anti-GPM6A antibody and MACS, the

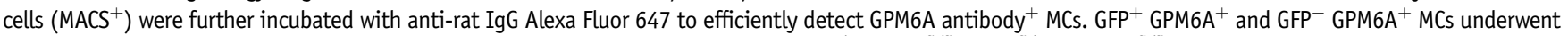
FACS from the fl/ + or fl/fl Tgfbr2 knockout mouse. MCs (Cre ${ }^{-}$MACS $^{+}$) isolated from $W t 1^{+/+} ; R_{26 T G^{f l} / f l} ;$ Tgfbr2 $^{\mathrm{fl} /+}$ or Tgfbr2 $^{\mathrm{fl} / \mathrm{fl}}$ mice and $\mathrm{Cre}^{+} \mathrm{MACS}^{-}$fractions were used

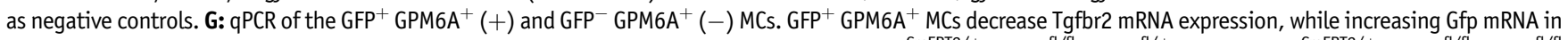
the homozygous (fl/fl) MCs. H: H\&E staining of the fibrotic body wall induced by CG from the $W t 1^{\mathrm{CreERT} 2 /+} ; R 26 \mathrm{TG}^{\mathrm{fl} / \mathrm{fl}} ; \mathrm{Tgfbr}^{\mathrm{fl} /+}$ (fl/ + ) or Wt1 ${ }^{\mathrm{CreERT} 2 /+} ; R 26 \mathrm{TG} \mathrm{G}^{\mathrm{fl} / \mathrm{fl}} ; \mathrm{Tgfbr}^{\mathrm{fl} / \mathrm{fl}}$ (fl/fl) mouse. I: Quantification of the area of connective tissue in $T g f b r 2^{\mathrm{fl} /+}$ and $T g f b r 2^{\mathrm{fl} / \mathrm{fl}}$ mice treated with $10 \mathrm{CG}$ injections in $\mathbf{H}$. J and $\mathrm{K}$ : Immunohistochemistry of the body wall from $\mathrm{Tgfbr}^{\mathrm{fl} /+}(\mathrm{fl} /+)$ or $T g f b r 2^{\mathrm{fl} / \mathrm{fl}}(\mathrm{fl} / \mathrm{fl})$ mice treated with $10 \mathrm{CG}$ injections with the use of anti-GFP antibodies (green) and ACTA2, GPM6A (J), or P-SMAD2/3 antibodies (red) (K). Arrowheads and arrows indicate MCs and myofibroblasts, respectively. J: The MC layer remains on the body wall after the conditional deletion of Tgfbr2 in MCs (an arrowhead). K: Double arrowheads indicate GFP ${ }^{+}$MCs without P-SMAD2/3 expression. Nuclei were counterstained with DAPI. $n=3$ (C and I). ${ }^{*} P<0.05,{ }^{*} P<0.01$. Scale bars: $100 \mu \mathrm{m}(\mathbf{B}$ and $\mathbf{H}) ; 10 \mu \mathrm{m}$ (D and $\left.\mathbf{K}\right)$. ACTA2, $\alpha$-smooth muscle actin; CG, chlorhexidine gluconate; FACS, fluorescence-activated cell sorter; GFP, green fluorescent protein; GPM6A, glycoprotein M6a; H\&E, hematoxylin and eosin; K0, knockout; MACS, magnetically activated cell sorting; MC, mesothelial cell; MMT, mesothelial-mesenchymal transition; P-SMAD3, phosphorylated-SMAD3; qPCR, quantitative real-time PCR; STR, soluble form of TGFBR2; TGF, transforming growth factor.
} 
A

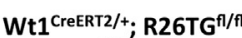

; $\mathrm{Tgfbr}^{\mathrm{f} / \mathrm{fl}}$ mouse

Tamoxifen x 2 (Tomato $\rightarrow$ GFP \& Tgfbr2 KO)

$\stackrel{\text { It }}{\underset{2 w k s}{\rightleftarrows} \uparrow \uparrow \uparrow_{\text {CG } \times 10}}$

C

Normal

$+/+$

$\mathrm{fl} / \mathrm{fl}$

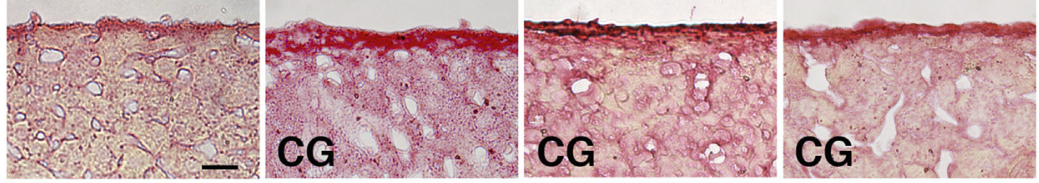

$+1$

$40 \times 48$

$\mathrm{CG}+4 \mathrm{~W}$

$C G+4 W$

E

$\mathrm{fl} / \mathrm{CG}$
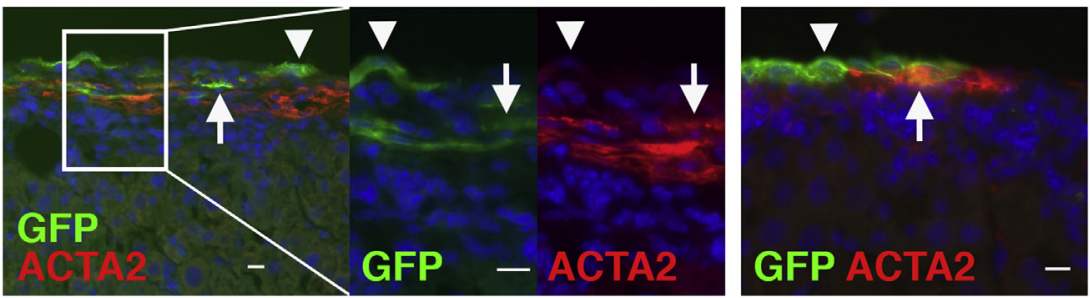

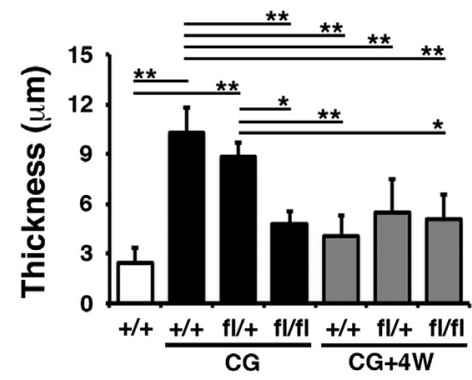

$\mathbf{F}$

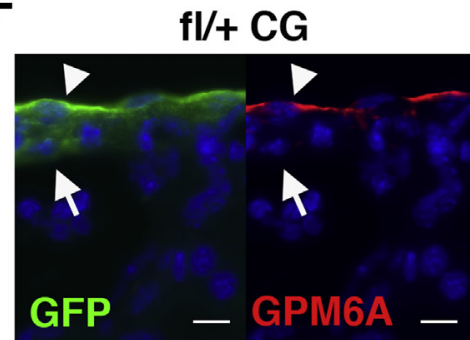

G

$\mathrm{fl} /+\mathrm{CG}$

fl/fl CG
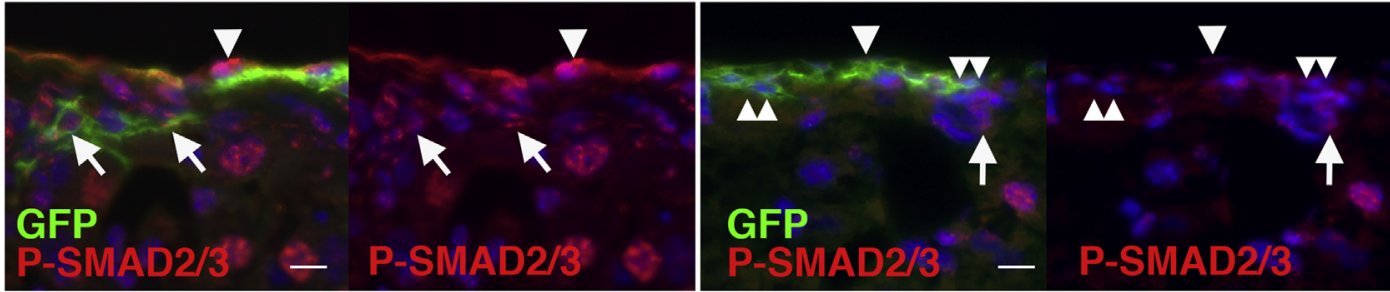

H

$\mathrm{fl} /+\mathrm{CG}+4 \mathrm{~W}$

fl/fl $C G+4 W$

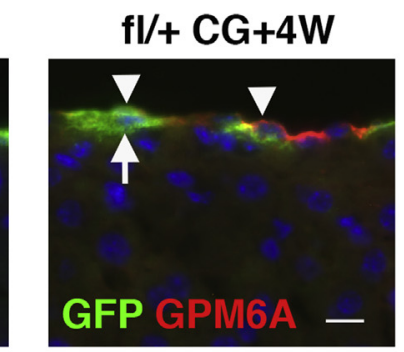

fl/fl CG+4W
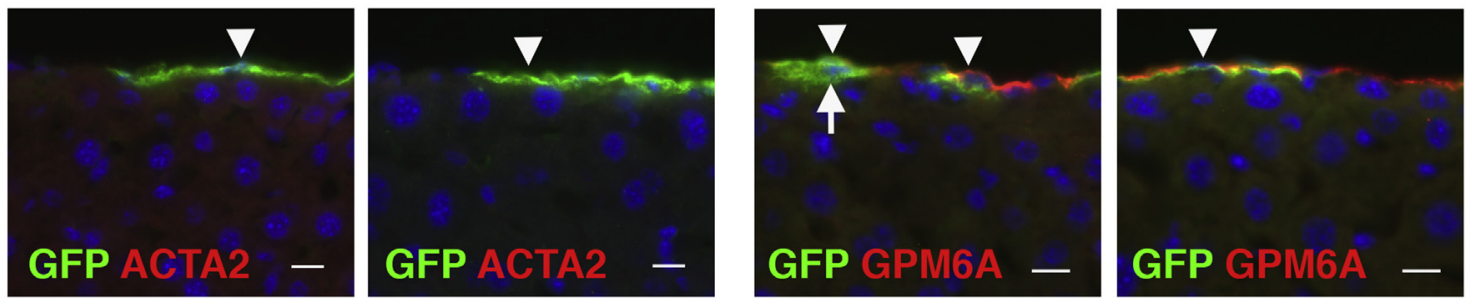

I

\section{J}

Body wall

MesP1 1 cre/+;

R26TG $^{\mathrm{I} / \mathrm{f}}$ mouse

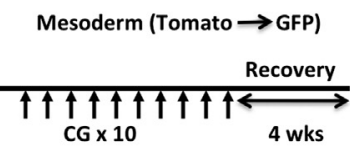
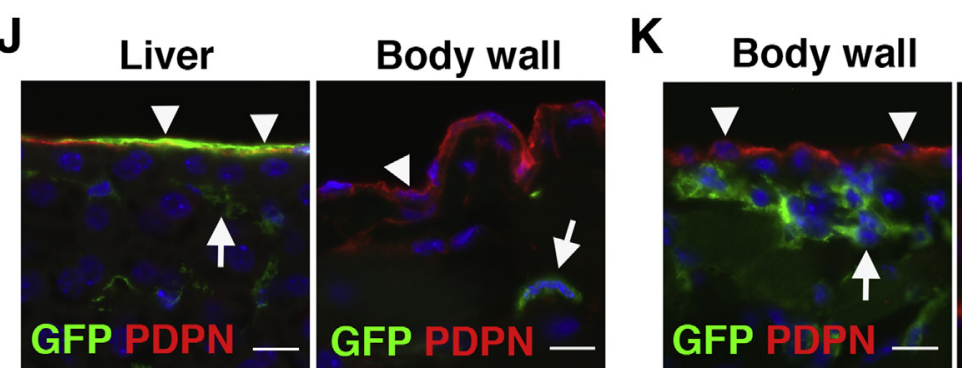

Body wall

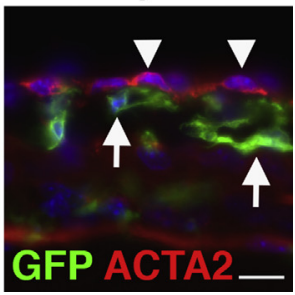


peritoneal fibrosis induced by TGF- $\beta{ }^{32}$ Canonical TGF- $\beta$ signaling is mediated by SMAD2/3. ${ }^{30}$ In MCs, SMAD3, but not SMAD2, mediates MMT. ${ }^{19,31}$ In addition to the canonical pathway, the TGF- $\beta /$ mammalian target of rapamycin pathway is also involved in MMT. ${ }^{19,30}$ In TGF- $\beta$-induced MMT, SNAIL1 was shown to down-regulate $\mathrm{CDH} 1$ expression and thereby induce MMT, similar to the mechanism of cancer metastasis. $^{20,30,33}$ However, we could not detect CDH1 expression in either peritoneal or liver MCs by immunohistochemistry in mice. ${ }^{12}$ Cultured MCs express Snail at a low level (data not shown). We assume that SNAIL1-mediated downregulation of CDH1 may not be involved in MMT in mice.

With the use of $W t 1^{\mathrm{CreERT} 2 /+} ; R 26 \mathrm{TG}^{\mathrm{ff} / \mathrm{fl}} ; T g f b r 2^{\mathrm{fl} / \mathrm{fl}}$ mice, we conditionally deleted $T g f b r 2$ in $W t 1^{+}$MCs. We visualized the migration and differentiation of Tgfbr2-null MCs as $\mathrm{GFP}^{+}$cells in the body wall. Compared with $T g f b r 2^{\mathrm{fl} /+}$ heterozygous mice, $T g f b r 2^{\mathrm{f} / \mathrm{fl}}$ homozygous knockout mice treated with CG showed few $\mathrm{GFP}^{+}$myofibroblasts in the connective tissue, indicating that direct TGF- $\beta$ signaling in MCs is essential for MMT. Although CreERT2-mediated recombination occurred only in $12.5 \%$ of MCs in our condition with the use of the $\mathrm{WtI}^{\mathrm{CreERT2}}$ mouse, the conditional $T g f b r 2$ knockout mouse showed the presence of mesothelium on the body wall and a reduction in the connective tissue area induced by $\mathrm{CG}$ injections, indicating that protecting the intact $\mathrm{MC}$ layer is beneficial in preventing peritoneal fibrosis.

We identified the expression of GPM6A, CD200, PDPN, UPK1B, KRT8, KRT19, VIM, and COL I in both peritoneal and liver MCs. GPM6A is a four-transmembrane domain protein and regulates neurite growth. ${ }^{34}$ Gpm6a-deficient mice develop normally but show abnormal behavior by stress. ${ }^{35}$ However, nothing is known about the function of GPM6A in MCs. We previously reported that liver MCs down-regulate mRNA and protein expression of GPM6A by TGF- $\beta$ treatment. ${ }^{12}$ Intriguingly, the present study showed that peritoneal MCs up-regulate Gpm6a mRNA expression by TGF- $\beta$ treatment, implying that the phenotype of peritoneal MCs is not identical to that of liver MCs. In fact, we found a distinct origin of peritoneal and liver MCs. At early embryogenesis, the lateral plate mesoderm is divided into the somatic and splanchnic mesoderm that gives rise to the parietal and visceral mesothelium, respectively. ${ }^{2}$ MESP1 is a basic helix-loop-helix transcription factor that is transiently expressed in the nascent mesoderm at the onset of gastrulation, and Mespl $^{+}$mesoderm contributes to a part of the lateral plate mesoderm. ${ }^{4}$ In the normal liver, Mesp $1^{+}$mesoderm gives rise to MCs and hepatic stellate cells that reside in the space of Disse. ${ }^{3,5}$ In liver fibrosis, both cells give rise to myofibroblasts. ${ }^{5}$ The present study showed that Mespl ${ }^{+}$mesoderm does not contribute to parietal MCs in mice, indicating distinct origins of MCs in the liver and body wall.

Peritoneal and liver MCs change phenotypes differently in CG-induced peritoneal fibrosis. With CG injection, peritoneal MCs on the body wall begin to express ACTA2 and differentiate into myofibroblasts. The MC layer largely disappears after repeated CG injections. However, liver MCs continue to cover the liver surface after CG injections and do not express ACTA2. Liver MCs give rise to $\mathrm{ACTA} 2^{+}$myofibroblasts that migrate beneath the MC layer, whereas the MC layer remains on the liver surface. Myofibroblasts derived from peritoneal MCs but not liver MCs express PDPN in the connective tissue. TGF- $\beta$ suppresses Gpm6a mRNA in the liver ${ }^{12}$ but not in peritoneal MCs. Our data indicate that peritoneal MCs and liver MCs are inherently different. Shelton et $\mathrm{a}^{36}$ reported that visceral MCs express autotaxin and have more migration capacity compared with parietal MCs. A recent study that used organ transplantation revealed that visceral MCs in the developing lung are derived from the lung but not from other organs. ${ }^{37}$ The intrinsic difference between parietal and visceral MCs may underlie the differential responses of liver and peritoneal MCs to injury caused by CG.

The peritoneal fibrosis induced by CG injections is reversible. ${ }^{38}$ In our model, the MC layer of the body wall is regenerated in 4 weeks after CG injections. Interestingly, regenerated MCs are negative for GFP in $\operatorname{Mespl}^{\mathrm{Cr} /+} ; R 26 \mathrm{TG}^{\mathrm{f} / \mathrm{fl}}$

\footnotetext{
Figure 6 Lineage tracing of liver and peritoneal MCs in CG-induced injury. A-H: Lineage tracing of MCs in the liver with the use of Wt1 ${ }^{\text {CreeRT2/+ }} ; R 26 \mathrm{TG} \mathrm{fl}^{\mathrm{fl} / \mathrm{fl}} ; \mathrm{Tgfbr}^{\mathrm{fl} / \mathrm{fl}}$

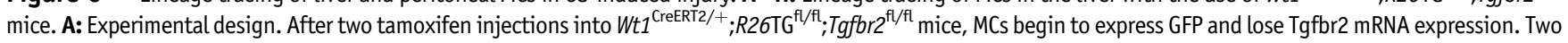
weeks after tamoxifen injection, the mice were treated with CG by 10 intraperitoneal injections. Four weeks after the last CG injection, the liver tissues were analyzed to examine the recovery. B: Immunohistochemistry of GFP (green) and GPM6A or ACTA2 (red) in the normal liver. GFP expression is observed in GPM6A ${ }^{+}$MCs (arrowhead) but not fibroblasts (arrow) beneath MCs. Liver MCs do not express ACTA2 (arrowheads). C: Sirius red staining of the liver after 10 CG injections (CG) or 4 weeks after the last CG injection (CG+4W). Sirius red staining shows thin collagen lamella beneath the liver surface. D: Quantification of the length of the collagen lamella in C. E-G: After labeling MCs as GFP ${ }^{+}$cells in $T g f b r 2^{\mathrm{fl} /+}$ or $T g f b r 2^{\mathrm{fl} / \mathrm{fl}}$ mice, liver injury was induced by $10 \mathrm{CG}$ injections. E: $\mathrm{GFP}^{+} \mathrm{ACTA}^{+}$myofibroblasts (arrows) are observed

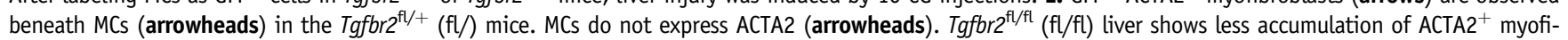
broblasts. F: After CG injections, MCs remain on the liver surface and express GPM6A (arrowheads). Arrows indicate GFP ${ }^{+}$MC-derived myofibroblasts beneath MCs. G: P-SMAD2/3 (red) is localized in the nuclei of MCs (arrowheads), myofibroblasts (arrows), and hepatocytes (large round nuclei) by CG treatment in the Tgfbr2 ${ }^{\mathrm{fl} /+}$ liver. $\mathrm{Tgfbr}^{\mathrm{fl} / \mathrm{fl}}$ liver shows down-regulation of P-SMAD2/3 in $\mathrm{GFP}^{+}$cells (double arrowheads). H: Four weeks after the last CG injection, the liver tissues were analyzed by immunohistochemistry for GFP (green) and ACTA2 or GPM6A (red) in $T g f b r 2^{\mathrm{fl} /+}$ or $\mathrm{Tgfbr}^{\mathrm{fl} / \mathrm{fl}}$ mice. GFP ${ }^{+}$MCs (arrowheads) are negative for ACTA2 and positive for GPM6A. An arrow indicates GFP ${ }^{+}$cells beneath MCs in regenerating liver. I-K: Mesoderm lineage tracing in the body wall and liver. I: Mesodermal lineage was traced with the use of Mesp $1^{\mathrm{Cre} /+} ;$;R26TG $\mathrm{G}^{\mathrm{fl} / \mathrm{fl}}$ mice. J: Normal liver and body wall tissues from the Mesp $1^{\mathrm{Cre} /+} ; R 26 \mathrm{TG} \mathrm{fl}^{\mathrm{fl} / \mathrm{l}}$ mouse were immunostained with GFP (green) and PDPN (red). Liver MCs, but not peritoneal MCs, express GFP (arrowheads). Arrows indicate GFP ${ }^{+}$hepatic stellate cells in the liver and fibroblasts in the body wall. K: Peritoneal fibrosis was induced by $10 \mathrm{CG}$ injections into Mesp $1^{\mathrm{Cre} /{ }_{+}^{+}} ; R 26 \mathrm{TG}^{\mathrm{fl} / \mathrm{fl}}$ mice, and the mice were allowed to recover for 4 weeks by discontinuing the $\mathrm{CG}$ treatment. The body wall tissues were immunostained with GFP (green) and PDPN or ACTA2 (red) 4 weeks after 10 CG injections. Note that no GFP expression is evident in regenerated peritoneal MCs (arrowheads). Arrows indicate fibroblasts expressing GFP in the connective tissue. Nuclei were counterstained with DAPI. $n=3$ (D); $n=4$ mice (I). ${ }^{*} P<0.05,{ }^{*} P<0.01$. Scale bars: $10 \mu \mathrm{m}(\mathbf{B}, \mathbf{E}-\mathbf{H}, \mathbf{J}$, and $\mathbf{K}) ; 20 \mu \mathrm{m}$ (C). ACTA2, $\alpha$-smooth muscle actin; CG, chlorhexidine gluconate; GFP, green fluorescent protein; GPM6A, glycoprotein M6a; K0, knockout; MC, mesothelial cell; P-SMAD3, phosphorylated-SMAD3; PDPN, podoplanin; 4W, 4 weeks.
} 
mice, refuting the possible contribution of liver MCs or body wall fibroblasts to the regenerated peritoneal MCs. After reversal from peritoneal fibrosis in $W t 1^{\mathrm{CreERT} 2 /+} ; R 26 \mathrm{TG}^{\mathrm{f} / / \mathrm{l}}$ mice, we found that $17.6 \%$ of peritoneal MCs express GFP, which is similar to the original labeling efficiency of peritoneal MCs (12.5\%) in this model, implying that surviving MCs are likely to be the source of regenerating MCs on the body wall. In fact, the BrdU incorporation assay revealed active DNA synthesis in MCs compared with myofibroblasts during recovery from peritoneal fibrosis. Another possible source is that MC-derived myofibroblasts revert to MCs during recovery. This hypothesis remains to be determined.

We found that $\mathrm{CG}$ also causes fibrosis under the liver surface. The liver surface is covered by a single layer of MCs, and capsular fibroblasts exist beneath MCs. ${ }^{39}$ Sirius red staining showed the presence of thin collagen lamella beneath MCs in normal mouse livers. After CG injections, collagen septa consisting of $\mathrm{ACTA}^{+}$myofibroblasts developed beneath the mesothelium. On the basis of the cell lineage tracing, we estimated that approximately $82 \%$ of ACTA $2^{+}$myofibroblasts are derived from MCs in CG-induced capsular fibrosis. Currently, there are no markers to characterize capsular fibroblasts in the liver, and their contribution to myofibroblasts in liver fibrosis remains unclear. Similar to peritoneal fibrosis, conditional deletion of $T g f b r 2$ gene in MCs suppressed the development of collagen septa and differentiation of MCs to myofibroblasts in CG-induced liver injury. These data indicate that TGF- $\beta$ signaling plays an important role in development of liver capsular fibrosis via induction of MMT.

In summary, the present study found that MCs partially contribute to myofibroblasts in peritoneal fibrosis. Conditional deletion of $T g f b r 2$ in $W t 1^{+}$MCs suppressed MMT and reduced peritoneal and liver fibrosis. The protection of MCs will be a therapeutic target for the prevention and suppression of peritoneal fibrosis.

\section{Supplemental Data}

Supplemental material for this article can be found at http://dx.doi.org/10.1016/j.ajpath.2015.08.009.

\section{References}

1. Mutsaers SE: The mesothelial cell. Int J Biochem Cell Biol 2004, 36 : $9-16$

2. Thomason RT, Bader DM, Winters NI: Comprehensive timeline of mesodermal development in the quail small intestine. Dev Dyn 2012, 241:1678-1694

3. Asahina K, Tsai SY, Li P, Ishii M, Maxson RE Jr, Sucov HM, Tsukamoto H: Mesenchymal origin of hepatic stellate cells, submesothelial cells, and perivascular mesenchymal cells during mouse liver development. Hepatology 2009, 49:998-1011

4. Saga Y, Miyagawa-Tomita S, Takagi A, Kitajima S, Miyazaki Ji, Inoue T: MesP1 is expressed in the heart precursor cells and required for the formation of a single heart tube. Development 1999, 126:3437-3447

5. Lua I, James D, Wang J, Wang KS, Asahina K: Mesodermal mesenchymal cells give rise to myofibroblasts, but not epithelial cells, in mouse liver injury. Hepatology 2014, 60:311-322
6. Que J, Wilm B, Hasegawa H, Wang F, Bader D, Hogan BL: Mesothelium contributes to vascular smooth muscle and mesenchyme during lung development. Proc Natl Acad Sci U S A 2008, 105:16626-16630

7. Zhou B, Ma Q, Rajagopal S, Wu SM, Domian I, Rivera-Feliciano J, Jiang D, von Gise A, Ikeda S, Chien KR, Pu WT: Epicardial progenitors contribute to the cardiomyocyte lineage in the developing heart. Nature 2008, 454:109-113

8. Asahina K, Zhou B, Pu WT, Tsukamoto H: Septum transversumderived mesothelium gives rise to hepatic stellate cells and perivascular mesenchymal cells in developing mouse liver. Hepatology 2011, 53:983-995

9. Winters NI, Thomason RT, Bader DM: Identification of a novel developmental mechanism in the generation of mesothelia. Development 2012, 139:2926-2934

10. Rinkevich Y, Mori T, Sahoo D, Xu PX, Bermingham JR Jr, Weissman IL: Identification and prospective isolation of a mesothelial precursor lineage giving rise to smooth muscle cells and fibroblasts for mammalian internal organs, and their vasculature. Nat Cell Biol 2012, 14:1251-1260

11. Dixit R, Ai X, Fine A: Derivation of lung mesenchymal lineages from the fetal mesothelium requires hedgehog signaling for mesothelial cell entry. Development 2013, 140:4398-4406

12. Li Y, Wang J, Asahina K: Mesothelial cells give rise to hepatic stellate cells and myofibroblasts via mesothelial-mesenchymal transition in liver injury. Proc Natl Acad Sci U S A 2013, 110:2324-2329

13. Karki S, Surolia R, Hock TD, Guroji P, Zolak JS, Duggal R, Ye T, Thannickal VJ, Antony VB: Wilms' tumor 1 (Wt1) regulates pleural mesothelial cell plasticity and transition into myofibroblasts in idiopathic pulmonary fibrosis. FASEB J 2014, 28:1122-1131

14. Hinz B, Phan SH, Thannickal VJ, Prunotto M, Desmoulière A, Varga J, De Wever O, Mareel M, Gabbiani G: Recent developments in myofibroblast biology: paradigms for connective tissue remodeling. Am J Pathol 2012, 180:1340-1355

15. Aroeira LS, Aguilera A, Sánchez-Tomero JA, Bajo MA, del Peso G, Jiménez-Heffernan JA, Selgas R, López-Cabrera M: Epithelial to mesenchymal transition and peritoneal membrane failure in peritoneal dialysis patients: pathologic significance and potential therapeutic interventions. J Am Soc Nephrol 2007, 18:2004-2013

16. Devuyst O, Margetts PJ, Topley N: The pathophysiology of the peritoneal membrane. J Am Soc Nephrol 2010, 21:1077-1085

17. Korte MR, Sampimon DE, Betjes MG, Krediet RT: Encapsulating peritoneal sclerosis: the state of affairs. Nat Rev Nephrol 2011, 7:528-538

18. Yáñez-Mó M, Lara-Pezzi E, Selgas R, Ramírez-Huesca M, Domínguez-Jiménez C, Jiménez-Heffernan JA, Aguilera A, SánchezTomero JA, Bajo MA, Alvarez V, Castro MA, del Peso G, Cirujeda A, Gamallo C, Sánchez-Madrid F, López-Cabrera M: Peritoneal dialysis and epithelial-to-mesenchymal transition of mesothelial cells. N Engl J Med 2003, 348:403-413

19. Patel P, Sekiguchi Y, Oh KH, Patterson SE, Kolb MR, Margetts PJ: Smad3-dependent and -independent pathways are involved in peritoneal membrane injury. Kidney Int 2010, 77:319-328

20. Strippoli R, Benedicto I, Foronda M, Perez-Lozano ML, SánchezPerales S, López-Cabrera M, Del Pozo MÁ: p38 maintains E-cadherin expression by modulating TAK1-NF-kappa B during epithelial-tomesenchymal transition. J Cell Sci 2010, 123:4321-4331

21. Xie JY, Chen N, Ren H, Wang WM: Angiotensin II-mediated activation of fibrotic pathways through ERK1/2 in rat peritoneal mesothelial cells. Ren Fail 2010, 32:871-879

22. Yokoi H, Kasahara M, Mori K, Ogawa Y, Kuwabara T, Imamaki H, Kawanishi T, Koga K, Ishii A, Kato Y, Mori KP, Toda N, Ohno S, Muramatsu H, Muramatsu T, Sugawara A, Mukoyama M, Nakao K: Pleiotrophin triggers inflammation and increased peritoneal permeability leading to peritoneal fibrosis. Kidney Int 2012, 81:160-169

23. Beavis MJ, Williams JD, Hoppe J, Topley N: Human peritoneal fibroblast proliferation in 3-dimensional culture: modulation by cytokines, growth factors and peritoneal dialysis effluent. Kidney Int 1997 , $51: 205-215$ 
24. Sakai N, Chun J, Duffield JS, Wada T, Luster AD, Tager AM: LPA1induced cytoskeleton reorganization drives fibrosis through CTGFdependent fibroblast proliferation. FASEB J 2013, 27:1830-1846

25. Chen YT, Chang YT, Pan SY, Chou YH, Chang FC, Yeh PY, Liu YH, Chiang WC, Chen YM, Wu KD, Tsai TJ, Duffield JS, Lin SL: Lineage tracing reveals distinctive fates for mesothelial cells and submesothelial fibroblasts during peritoneal injury. J Am Soc Nephrol 2014, 25:2847-2858

26. Yata Y, Scanga A, Gillan A, Yang L, Reif S, Breindl M, Brenner DA, Rippe RA: DNase I-hypersensitive sites enhance alpha1(I) collagen gene expression in hepatic stellate cells. Hepatology 2003, 37: $267-276$

27. Muzumdar MD, Tasic B, Miyamichi K, Li L, Luo L: A global doublefluorescent Cre reporter mouse. Genesis 2007, 45:593-605

28. Levéen P, Larsson J, Ehinger M, Cilio CM, Sundler M, Sjöstrand LJ, Holmdahl R, Karlsson S: Induced disruption of the transforming growth factor beta type II receptor gene in mice causes a lethal inflammatory disorder that is transplantable. Blood 2002, 100:560-568

29. Kushiyama T, Oda T, Yamada M, Higashi K, Yamamoto K, Oshima N, Sakurai Y, Miura S, Kumagai H: Effects of liposomeencapsulated clodronate on chlorhexidine gluconate-induced peritoneal fibrosis in rats. Nephrol Dial Transplant 2011, 26:3143-3154

30. Derynck R, Muthusamy BP, Saeteurn KY: Signaling pathway cooperation in TGF- $\beta$-induced epithelial-mesenchymal transition. Curr Opin Cell Biol 2014, 31:56-66

31. Duan WJ, Yu X, Huang XR, Yu JW, Lan HY: Opposing roles for Smad2 and Smad3 in peritoneal fibrosis in vivo and in vitro. Am J Pathol 2014, 184:2275-2284
32. Motomura Y, Kanbayashi H, Khan WI, Deng Y, Blennerhassett PA, Margetts PJ, Gauldie J, Egashira K, Collins SM: The gene transfer of soluble VEGF type I receptor (Flt-1) attenuates peritoneal fibrosis formation in mice but not soluble TGF-beta type II receptor gene transfer. Am J Physiol Gastrointest Liver Physiol 2005, 288:G143-G150

33. Zhou Q, Yang M, Lan H, Yu X: miR-30a negatively regulates TGF$\beta 1$-induced epithelial-mesenchymal transition and peritoneal fibrosis by targeting Snai1. Am J Pathol 2013, 183:808-819

34. Alfonso J, Fernandez ME, Cooper B, Flugge G, Frasch AC: The stressregulated protein M6a is a key modulator for neurite outgrowth and filopodium/spine formation. Proc Natl Acad Sci U S A 2005, 102: $17196-17201$

35. El-Kordi A, Kästner A, Grube S, Klugmann M, Begemann M, Sperling S, Hammerschmidt K, Hammer C, Stepniak B, Patzig J, de Monasterio-Schrader P, Strenzke N, Flügge G, Werner HB, Pawlak R, Nave KA, Ehrenreich H: A single gene defect causing claustrophobia. Transl Psychiatry 2013, 3:e254

36. Shelton EL, Galindo CL, Williams CH, Pfaltzgraff E, Hong CC, Bader DM: Autotaxin signaling governs phenotypic heterogeneity in visceral and parietal mesothelia. PLoS One 2013, 8:e69712

37. Winters NI, Williams AM, Bader DM: Resident progenitors, not exogenous migratory cells, generate the majority of visceral mesothelium in organogenesis. Dev Biol 2014, 391:125-132

38. Nishioka Y, Miyazaki M, Abe K, Furusu A, Harada T, Ozono Y, Taguchi T, Koji T, Kohno S: Regeneration of peritoneal mesothelium in a rat model of peritoneal fibrosis. Ren Fail 2008, 30:97-105

39. Bhunchet E, Wake K: Role of mesenchymal cell populations in porcine serum-induced rat liver fibrosis. Hepatology 1992, 16:1452-1473 